TRANSACTIONS OF THE

AMERICAN MATHEMATICAL SOCIETY

Volume 365, Number 7, July 2013, Pages 3413-3436

S 0002-9947(2013)05754-6

Article electronically published on January 30, 2013

\title{
ON QUANDLE HOMOLOGY GROUPS OF ALEXANDER QUANDLES OF PRIME ORDER
}

\author{
TAKEFUMI NOSAKA
}

\begin{abstract}
In this paper we determine the integral quandle homology groups of Alexander quandles of prime order. As a special case, this settles the delayed Fibonacci conjecture by M. Niebrzydowski and J. H. Przytycki from their 2009 paper. Further, we determine the cohomology group of the Alexander quandle and obtain relatively simple presentations of all higher degree cocycles which generate the cohomology group. Finally, we prove that the integral quandle homology of a finite connected Alexander quandle is annihilated by the order of the quandle.
\end{abstract}

\section{INTRODUCTION}

A quandle is a set with a certain binary operation: the operation is, roughly speaking, defined under some weaker conditions than groups. In analogy to geometric realizations of group (co)homology, classifying spaces of quandles were first introduced by R. Fenn, C. Rourke and B. Sanderson in FRS. As a modification, the quandle (co)homology of a quandle $X$ is defined by J. S. Carter, D. Jelsovsky, S. Kamada, L. Langford and M. Saito in CJKLS. The 2,3 or 4-cocycles of the quandle cohomology give rise to the quandle cocycle invariants for 1-knots and 2-knots (see CJKLS, CKS or O] for details). Further, concerning higher dimensional knots, J. S. Carter, S. Kamada and M. Saito in CKS] investigated the correspondence between some higher dimensional link-diagrams colored by $X$ and some cycles of quandle homology; the pairings between these higher degree cycles and cocycles are expected to be invariants of higher dimensional links of codimension two. Therefore, for the study of the invariants of links or of the pairings, it is important to find explicit formulae of quandle cocycles and to determine quandle cohomology groups.

However, it is not so easy to calculate quandle (co)homology groups, since there are fewer methods to study them than group (co)homology theory. But some results on quandle (co)homologies are known: T. Mochizuki listed all 2-cocycles for finite Alexander quandles over a finite field in [M1] and all 3-cocycles for Alexander quandles on a finite field in M2]. A presentation of a 3-cocycle found in M1 gives rise to some applications for 2-knots (see $\mathrm{AS}$ ] for example). R. A. Litherland and S. Nelson analyzed the free and torsion subgroup of the quandle homology group of a finite quandle in [LN]. Regarding quandle homologies of higher degree, M. Niebrzydowski and J. H. Przytycki constructed some quandle homological operations and

Received by the editors November 17, 2009 and, in revised form, April 1, 2011.

2010 Mathematics Subject Classification. Primary 20G10, 55N35, 58H10; Secondary 57Q45, 57M25, 55S20.

Key words and phrases. Rack, quandle, homology, cohomology, knot. 
estimated the torsion subgroups of the integral quandle homology groups of some quandles in $\mathrm{NP}$.

In this paper we determine the integral quandle homology groups of all Alexander quandles of prime order $p$. Here this quandle is defined to be $\mathbb{Z}_{p}$ with a binary operation given by $x * y=\omega x+(1-\omega) y$, where $\mathbb{Z}_{p}$ means a cyclic group of order $p$ and $\omega \in \mathbb{Z}_{p}$ is neither 0 nor 1$]$ The class of the quandles on $\mathbb{Z}_{p}$ is known to be the simplest non-trivial class among quandles. To be specific, it is known EGS that any "connected" quandle of order $p$ is isomorphic to one of the Alexander quandles. We denote the integral quandle homology by $H_{n}^{Q}(X ; \mathbb{Z})$. Our main result is to determine $H_{n}^{Q}(X ; \mathbb{Z})$ of all quandles of the simplest class as follows:

Theorem 1.1. Let $X$ be the Alexander quandle of order $p$ with $\omega \neq 0,1$. Then the integral quandle homology groups are $H_{1}^{Q}(X ; \mathbb{Z}) \cong \mathbb{Z} \oplus \mathbb{Z}_{p}^{b_{1}}$ and $H_{n}^{Q}(X ; \mathbb{Z}) \cong \mathbb{Z}_{p}^{b_{n}}$ for $n \geq 2$, where $b_{n}$ is determined by

$$
b_{n+2 e}=b_{n}+b_{n+1}+b_{n+2}, \quad b_{1}=b_{2}=\cdots=b_{2 e-2}=0, \quad \text { and } b_{2 e-1}=b_{2 e}=1,
$$

and $e$ is the order of $\omega$, that is, $e>0$ is the minimal number satisfying $\omega^{e}=1$.

We discuss some corollaries. Theorem 1.1 shows that $H_{n}^{Q}(X ; \mathbb{Z}) \cong 0$ for the Alexander quandle $X$ with $\omega \neq-1,0,1$ and $2 \leq n \leq 4$ (Corollary 2.3), since $e>2$ and $2 e-1>4$. Therefore, this tells us that only the case $\omega=-1$ is useful in Alexander quandles of prime order for the study of quandle cocycle invariants of 1-knots and 2-knots. Furthermore, when $\omega=-1$, the Alexander quandle $X$ is said to be a dihedral quandle. As a corollary of Theorem 1.1, we settle the delayed Fibonacci conjecture by M. Niebrzydowski and J. H. Przytycki [NP] (see Corollary 2.2): they conjectured that the quandle homologies $H_{n}^{Q}(X ; \mathbb{Z})$ of dihedral quandles are characterized by a "delayed Fibonacci sequence".

Besides, we discuss the torsion subgroup of $H_{n}^{Q}(M ; \mathbb{Z})$. Here we deal with, more generally, connected Alexander quandles $M$ of finite order: the quandle is defined by a $\mathbb{Z}\left[T^{ \pm}\right]$-module $M$ satisfying $(1-T) M=M$ with a binary operation given by $x * y=T x+(1-T) y$. A remarkable result is that $H_{n}^{Q}(M ; \mathbb{Z})$ is annihilated by $|M|$ for $n \geq 2$ (Corollary 6.2). As a special case, if $X$ is the Alexander quandle of order $p$ with $\omega \in \mathbb{Z}_{p}$, then $H_{n}^{Q}(X ; \mathbb{Z})$ is annihilated by $p$ (Corollary 6.4, which settles $\left[\mathrm{NP}\right.$, Conjecture 16]). It is known $\left[\mathrm{LN}\right.$, Theorem 1] that $H_{n}^{Q}(X ; \mathbb{Z})$ is annihilated by $|X|^{n}$ for a connected quandle $X$ with a certain condition. Namely, Corollary 6.2 is a stronger estimate for Alexander quandles.

Corollary 6.2 also plays a role in the proof of Theorem 1.1. Let $X$ be the Alexander quandle of order $p$. Since $H_{n}^{Q}(X ; \mathbb{Z})$ is annihilated by $p$ as above, it is a $\mathbb{Z}_{p}$-vector space; hence, to show Theorem [1.1, it suffices to determine the dimension of the space. To determine the dimension, we will compute that of the quandle cohomologies, since it is easier to deal with cohomologies than homologies in general. We give a more detailed outline of the computations in Section 2.3, and note that our proof follows from a generalization of the Mochizuki approach to the third cohomologies in M1, M2.

\footnotetext{
${ }^{1}$ We ignore the case of $\omega=0$ and 1 throughout this paper. If $\omega=0$, then the binary operation is forbidden by quandle axioms. When $\omega=1$, the Alexander quandle is a trivial quandle. Hence we can easily obtain the quandle homology: $H_{n}^{Q}(X ; \mathbb{Z}) \cong \mathbb{Z}^{p(p-1)^{n-1}}$. We thus omit the case $p=2$.
} 
Our approach to proving our theorem provides benefits on quandle cocycles. More concisely, we determine the quandle cohomolgies (Theorem [3.3), and obtain simple presentations of all higher degree cocycles (Corollary [5.8): we write down explicitly the presentations in Examples 5.1, 5.2 and 5.3. A key to obtaining the cocycles is an operation

$$
\bar{\Omega}_{n} \oplus \bar{\Omega}_{n-1}: H_{Q}^{n}\left(X ; \mathbb{Z}_{p}\right) \oplus H_{Q}^{n+1}\left(X ; \mathbb{Z}_{p}\right) \oplus H_{Q}^{n+2}\left(X ; \mathbb{Z}_{p}\right) \longrightarrow H_{Q}^{n+2 e}\left(X ; \mathbb{Z}_{p}\right),
$$

where $e$ is the order of $\omega$. The operation is shown to be isomorphic (Corollary 5.6 and Remark 5.7); hence, presentations of all higher degree cocycles are obtained from those of lower degree via the operation by induction on $n$ as desired. We present concretely the 4-cocycles for use in Example 5.9. These presentations of all quandle cocycles are expected to apply to invariants of higher dimensional links.

In a future paper [No], the author will discuss the 4-cocycles obtained above. Unfortunately, the quandle cocycle invariants using the 4-cocycles of linked surfaces are equal to those using the 3-cocycle [No, Corollary 3.8]. On the other hand, recently, Clauwens [Cla] calculated the quandle homology of the dihedral quandle by algebraic topology.

This paper is organized as follows. Section 2 reviews the quandle homology and reformulates Theorem 1.1. Section 3 contains preliminaries for the proof. Section 4 introduces some isomorphisms and calculates $H_{Q}^{n}\left(X ; \mathbb{Z}_{p}\right)$ for $n \leq 2 e-1$. Section 5 determines all the quandle cohomology, leading to Theorem 1.1 Section 6 discusses the torsion parts of the quandle homologies for connected Alexander quandles.

\section{Results}

Throughout this paper we fix an odd prime number $p$. We review quandle homologies of Alexander quandles in Section 2.1. We state the main result (Theorem 2.1) in Section 2.2 and outline the proof in Section 2.3

2.1. Preliminaries: Rack and quandle homology groups. We begin by reviewing Alexander quandles, which are an interesting class of quandles in this paper. An Alexander quandle is defined to be a $\mathbb{Z}\left[T^{ \pm}\right]$-module with a binary operation given by $x * y=T x+(1-T) y$. It is known EGS that any "connected" quandle of prime order is isomorphic to an Alexander quandle of type $\mathbb{Z}\left[T^{ \pm}\right] /(p, T-\omega)$ for some $\omega \in \mathbb{Z}_{p}$, where $\omega$ is neither 0 nor 1 (see also [O, Section 5.1]). As it were, this type is the simplest non-trivial quandle among quandles. If $\omega=-1$, the Alexander quandle is called a dihedral quandle.

We next review the rack and quandle homology groups introduced in CJKLS. Given an Alexander quandle $(X, *)$ and a commutative ring $A$, let $C_{n}^{R}(X ; A)$ be the free $A$-module generated by $n$-tuples $\left(x_{1}, x_{2}, \ldots, x_{n}\right)$ of $X^{n}$; in other words $C_{n}^{R}(X ; A)=A\left\langle X^{n}\right\rangle$. Define its boundary homomorphism $\partial_{n}^{R}: C_{n}^{R}(X ; A) \rightarrow$ $C_{n-1}^{R}(X ; A)$ for $n \geq 2$ to be

$$
\begin{gathered}
\partial_{n}^{R}\left(x_{1}, \ldots, x_{n}\right)=\sum_{2 \leq i \leq n}(-1)^{i}\left(\left(x_{1} * x_{i}, \ldots, x_{i-1} * x_{i}, x_{i+1}, \ldots, x_{n}\right)\right. \\
\left.-\left(x_{1}, \ldots, x_{i-1}, x_{i+1}, \ldots, x_{n}\right)\right),
\end{gathered}
$$

and $\partial_{1}^{R}$ to be the zero map. The composite $\partial_{n-1}^{R} \circ \partial_{n}^{R}$ is known to be zero. The rack complex of $X$ is the pair $\left(C_{*}^{R}(X ; A), \partial_{*}^{R}\right)$. Furthermore, since $x * x=x$ for any $x \in X$, we have a subcomplex $C_{n}^{D}(X ; A) \subset C_{n}^{R}(X ; A)$ generated by $n$ tuples $\left(x_{1}, \ldots, x_{n}\right)$ with $x_{i}=x_{i-1}$ for some $i$. Then we call the quotient complex 
$C_{n}^{Q}(X ; A)=C_{n}^{R}(X ; A) / C_{n}^{D}(X ; A)$ the quandle complex of $X$. We denote the homologies of these complexes by $H_{n}^{R}(X ; A), H_{n}^{D}(X ; A)$, and $H_{n}^{Q}(X ; A)$, respectively. This $H_{n}^{R}(X ; A)$ is called the rack homology, and $H_{n}^{Q}(X ; A)$ is called the quandle homology.

We recall known results on the quandle homologies of Alexander quandles $X$ with $\omega$ of order $p$. By direct calculation it can be verified that $H_{1}^{R}(X ; \mathbb{Z}) \cong \mathbb{Z}$ and $H_{1}^{Q}(X ; \mathbb{Z}) \cong \mathbb{Z}$ (see also [LN]). T. Mochizuki showed [M1, Corollary 2.2] $H_{2}^{Q}(X ; \mathbb{Z}) \cong 0$ and proved [M1, Theorem 3.1] that $H_{3}^{Q}(X ; \mathbb{Z}) \cong 0$ if $\omega \neq-1,0,1$. Also, in the case $\omega=-1$, M. Niebrzydowski and J. Przytycki showed in NP] that $H_{3}^{Q}(X ; \mathbb{Z}) \cong \mathbb{Z}_{p}$ and $\mathbb{Z}_{p} \subset H_{4}^{Q}(X ; \mathbb{Z})$. It is also known [NP, Corollary 10] that $H_{n}^{Q}(X ; \mathbb{Z})$ is annihilated by $p^{n-2}$.

2.2. Main results. We will state our main theorem and give some corollaries and examples. We determine the integral quandle homology group of every Alexander quandle of order $p$ as follows.

Theorem 2.1. Let $\omega \in \mathbb{Z}_{p}$ be $\omega \neq 0,1$. Let $X$ be an Alexander quandle of type $X=\mathbb{Z}[T] /(p, T-\omega)$. Then the integral quandle homology groups are $H_{n}^{Q}(X ; \mathbb{Z}) \cong$ $\mathbb{Z}_{p}^{b_{n}}$ for $n \geq 2$ and $H_{1}^{Q}(X ; \mathbb{Z}) \cong \mathbb{Z} \oplus \mathbb{Z}_{p}^{b_{1}}$, where $b_{n}$ is determined by $b_{n}=b_{n-2 e}+$ $b_{n-2 e+1}+b_{n-2 e+2}, b_{1}=b_{2}=\cdots=b_{2 e-2}=0$, and $b_{2 e-1}=b_{2 e}=1$, and $e$ is the order of $\omega$. In other words, $e$ is the minimal number satisfying $\omega^{e}=1$.

As a special case, we obtain the homology in the case $\omega=-1$. This settles the delayed Fibonacci conjecture by M. Niebrzydowski and J. H. Przytycki [NP, Conjecture 5]:

Corollary 2.2 ([NP, Conjecture 5]). Let $X$ be the dihedral quandle of order $p$. Then $H_{n}^{Q}(X ; \mathbb{Z}) \cong \mathbb{Z}_{p}^{b_{n}}$ for $n \geq 2$ and $H_{1}^{Q}(X ; \mathbb{Z}) \cong \mathbb{Z} \oplus \mathbb{Z}_{p}^{b_{1}}$, where $b_{n}$ is determined by $b_{n+3}=b_{n+2}+b_{n}, b_{1}=b_{2}=0$, and $b_{3}=1$.

Proof. Note that $e=2$. Put $F_{b}(x)=\sum_{i \geq 1} b_{i} x^{i} \in \mathbb{Z}[[x]]$. Theorem 2.1 says

$$
F_{b}(x)=\frac{b_{1} x^{1}+b_{2} x^{2}+b_{3} x^{3}+b_{4} x^{4}}{1-x^{2}-x^{3}-x^{4}}=\frac{x^{3}+x^{4}}{1-x^{2}-x^{3}-x^{4}}=\frac{x^{3}}{1-x-x^{3}} .
$$

Therefore, the generating function leads to the condition as required.

For the study of quandle cocycle invariants of 1-knots and 2-knots, it is important to determine $H_{n}^{Q}(X ; \mathbb{Z})$ for $n=2,3$ or 4 (see [CJKLS] and [CKS] for details). It follows from the next corollary that only the dihedral quandle is useful in Alexander quandles of prime order for the invariants.

Corollary 2.3. Let $X$ be an Alexander quandle of order $p$ with $\omega \neq-1,0,1$. Then $H_{n}^{Q}(X ; \mathbb{Z}) \cong 0$ for $n=2,3$ or 4 .

Proof. Since $\omega \neq-1$, we see $2 e-1>4$.

By Corollaries 2.2 and 2.3, the Betti number of the homology $H_{n}^{Q}(X ; \mathbb{Z})$ in the case of $n \leq 4$ or $\omega=-1$ does not depend on the odd prime $p$. However, the higher degree homology groups with $\omega \neq-1$ do depend on $p$ and $\omega$ in general. 
2.3. Outline of the proof. Here we outline the proof of Theorem 2.1. Let $X$ be an Alexander quandle of order $p$. As we will show later on, $H_{n}^{Q}(X ; \mathbb{Z})$ is annihilated by $p$ (Corollary 6.4). This implies that $H_{n}^{Q}(X ; \mathbb{Z})$ is a finite dimensional $\mathbb{Z}_{p}$-vector space, i.e., $H_{n}^{Q}(X ; \mathbb{Z}) \cong \mathbb{Z}_{p}^{b_{n}}$ for some $b_{n}$. Hence, if we know the dimension of $H_{n}^{Q}(X ; \mathbb{Z})$, then the proof of Theorem 2.1 would be complete. For this we will calculate the quandle "cohomology" group with $\mathbb{Z}_{p}$-coefficient.

The outline for studying the cohomology $H_{Q}^{n}\left(X ; \mathbb{Z}_{p}\right)$ is a generalization of Mochizuki's approach [M2] to $H_{Q}^{k}\left(X ; \mathbb{Z}_{p}\right)$ for $k \leq 3$. Similarly to [M2, Section 2], we first give a decomposition of $H_{Q}^{n}\left(X ; \mathbb{Z}_{p}\right)$ in Section 3 . We will define another cohomology $H^{n(0)}(X)$ given by (4) , and show that $H_{Q}^{n}\left(X ; \mathbb{Z}_{p}\right) \cong H^{n(0)}(X) \oplus H^{n-1(0)}(X)$ for each $n \geq 2$ (Proposition 3.2). As a corollary, by the universal coefficient theorem, we will show $b_{n}=c_{n}^{(0)}$ for $n \geq 2$ (Lemma 5.5) , where $c_{n}^{(0)} \operatorname{denotes} \operatorname{dim}\left(H^{n(0)}(X)\right)$. Therefore, we shall turn to the study of the dimension of $H^{n(0)}(X)$. For the study on $H^{n(0)}(X)$, we will construct an isomorphism $\bar{\phi}$ from $H^{n(0)}(X)$ to a quotient vector space (Proposition 3.7). This quotient is a slight modification of a quotient vector space introduced in [M2, Section 3.2.4], where the third cohomology was reduced to an analysis of the quotient space.

However, we need further steps to determine $H^{n(0)}(X)$ for $n \geq 4$, as contrasted with $n \leq 3$. To begin, we set up some other quotient vector spaces in Section 4.1 . Using them, a key step is to construct the following composite of some isomorphisms:

$$
\begin{aligned}
& \bar{\phi}^{-1} \circ\left(\bar{\Theta}_{1}\right)^{-1} \circ \cdots \circ\left(\bar{\Theta}_{e-1}\right)^{-1} \circ \bar{\Psi}^{m}: H^{m-4(0)}(X) \oplus H^{m-3(0)}(X) \oplus H^{m-2(0)}(X) \\
& \longrightarrow H^{n(0)}(X),
\end{aligned}
$$

where $m=n-2 e+4$. In more detail, in Section 4.1 we will construct the map $\bar{\Theta}_{i}$ given by (15) and show that the map $\bar{\Theta}_{i}$ is isomorphic (Proposition 4.2). Further, we will define $\bar{\Psi}^{m}$ by (29) in Section [5.1) and show that $\bar{\Psi}^{m}$ is an isomorphism in Section 5.2

After the constructions, the above isomorphisms tell us that $c_{n}^{(0)}=c_{n-2 e}^{(0)}+$ $c_{n-2 e+1}^{(0)}+c_{n-2 e+2}^{(0)}$. On the other hand, we show that $c_{1}^{(0)}=c_{2}^{(0)}=\cdots=c_{2 e-2}^{(0)}=$ $0, c_{2 e-1}^{(0)}=c_{2 e}^{(0)}=1$ in Section 4.3. Since $b_{n}=c_{n}^{(0)}$ as mentioned above, this completes the proof of Theorem 2.1.

Here we remark on presentations of all $n$-cocycles of $H^{n(0)}(X)$. The above isomorphisms are constructed by polynomial segments (see Propositions 3.10 and 5.4 , and Corollary 4.4). Then we obtain relatively simple presentations of all higher degree cocycles which generate $H^{n(0)}(X)$ from those of lower degree cocycles (Corollary (5.8).

\section{Quandle cohomology groups of Alexander quandles}

As mentioned in Section 2.3, for the proof of Theorem 2.1, we shall investigate the quandle cohomologies. In Section 3.1, we reformulate quandle cochain groups and decompose the groups. In Section 3.2 we state Theorem 3.3 determining the cohomology. In Section 3.3 we prepare a differential operation and an integral operation on the cochain group. These operations play a fundamental role in this paper. In Section 3.4 for the search of $H^{n(0)}(X)$, we will construct an isomorphism from $H^{n(0)}(X)$ to a quotient space (Proposition 3.7). 
3.1. Preliminaries: A reformulation of quandle cochain groups. For calculations of quandle (co)homology groups of Alexander quandles, it is convenient to change another "coordinate system" such as in M2. There is not anything new in this subsection. For an Alexander quandle $M$, we define $C_{n}^{R_{U}}(M ; A)$ to be the free $A$-module generated by $n$-tuples $\left(U_{1}, \ldots, U_{n}\right)$ of $M^{n}$ and define the boundary map by

$$
\partial_{n}\left(U_{1}, \ldots, U_{n}\right)=\sum_{1 \leq i \leq n-1}(-1)^{i}\left(\left(T \cdot U_{1}, \ldots, T \cdot U_{i-1}, T \cdot U_{i}+U_{i+1}, U_{i+2}, \ldots, U_{n}\right)\right.
$$

$$
\left.-\left(U_{1}, \ldots, U_{i-1}, U_{i}+U_{i+1}, U_{i+2}, \ldots, U_{n}\right)\right),
$$

for $n \geq 2$, and $\partial_{1}$ to be the zero map. It can be verified that $\partial_{n-1} \circ \partial_{n}=0$. Further we have a subcomplex group generated by $n$-tuples $\left(U_{1}, \ldots, U_{n}\right)$ with $U_{i}=0$ for some $1 \leq i \leq n-1$. Then we have the quotient complex denoted by $C_{n}^{Q_{U}}(M ; A)$. Dually, we can define the cochain groups denoted by $C_{R_{U}}^{n}(M ; A)$ and $C_{Q_{U}}^{n}(M ; A)$.

We will give a canonical correspondence between these two complexes of Alexander quandles. Let us consider a bijection from $M^{n}$ to $M^{n}$ given by

$$
M^{n} \ni\left(x_{1}, \ldots, x_{n}\right) \mapsto\left(x_{1}-x_{2}, x_{2}-x_{3}, \ldots, x_{n-1}-x_{n}, x_{n}\right) \in M^{n} .
$$

The map induces an isomorphism $C_{n}^{R}(M ; A)\left(\operatorname{resp} . C_{n}^{Q}(M ; A)\right)$ to $C_{n}^{R_{U}}(M ; A)$ (resp. $\left.C_{n}^{Q_{U}}(M ; A)\right)$. Moreover, these isomorphisms are easily verified to be chain maps. We will mainly deal with the complexes $C_{n}^{R_{U}}(M ; A)$ and $C_{n}^{Q_{U}}(M ; A)$ in Sections 3 to 6 .

We will review a reformulation of the quandle cochain groups used in [M2]. Let $X$ be an Alexander quandle of order $p$ with $\omega \in \mathbb{Z}_{p}$ in all of the following. For $n \geq 1$, we define

$$
\begin{aligned}
C^{n}(X)=\left\{\sum a_{i_{1}, \ldots, i_{n}} \cdot U_{1}^{i_{1}} \cdots U_{n}^{i_{n}} \in \mathbb{Z}_{p}\left[U_{1}, \ldots, U_{n}\right] \mid\right. \\
\left.1 \leq i_{j} \leq p-1(j \leq n-1), i_{n} \leq p-1\right\},
\end{aligned}
$$

and $C^{0}(X)=\mathbb{Z}_{p}$. The coboundary is defined as follows: for $f \in C^{n}(X)$ and $n \geq 1$,

$$
\begin{aligned}
\delta_{n}(f) & \left(U_{1}, \ldots, U_{n+1}\right) \\
:= & \sum_{1 \leq i \leq n}(-1)^{i-1}\left(f\left(\omega \cdot U_{1}, \ldots, \omega \cdot U_{i-1}, \omega \cdot U_{i}+U_{i+1}, U_{i+2}, \ldots, U_{n+1}\right)\right. \\
& \left.-f\left(U_{1}, \ldots, U_{i-1}, U_{i}+U_{i+1}, U_{i+2}, \ldots, U_{n+1}\right)\right) \in \mathbb{Z}_{p}\left[U_{1}, \ldots, U_{n+1}\right],
\end{aligned}
$$

and $\delta_{0}$ is a zero map. We can check that $\delta_{n}\left(C^{n}(X)\right) \subset C^{n+1}(X)$ and $\delta_{n+1} \circ \delta_{n}=0$ for any $n$. The cohomology group is denoted by $H^{n}(X)$. The complex $C^{n}(X)$ is isomorphic to the cochain group $C_{Q_{U}}^{n}\left(X ; \mathbb{Z}_{p}\right)$ in Section 2. Hence, the universal coefficient theorem leads to

$$
H^{n}(X) \cong \operatorname{Hom}\left(H_{n}^{Q_{U}}(X ; \mathbb{Z}), \mathbb{Z}_{p}\right) \oplus \operatorname{Ext}^{1}\left(H_{n-1}^{Q_{U}}(X ; \mathbb{Z}), \mathbb{Z}_{p}\right) .
$$

We will decompose the complex $C^{n}(X)$ by the homogenous degree, that is,

$$
C_{d}^{n}(X):=\left\{\sum a_{i_{1}, \ldots, i_{n}} \cdot U_{1}^{i_{1}} \cdots U_{n}^{i_{n}} \in C^{n}(X) \mid \sum_{1 \leq k \leq n} i_{k}=d\right\} .
$$

Since $\delta_{n}\left(C_{d}^{n}(X)\right) \subset C_{d}^{n+1}(X)$, we obtain a direct sum decomposition of the complexes as $\left(C^{n}(X), \delta_{n}\right)=\left(\bigoplus_{d} C_{d}^{n}(X), \delta_{n}\right)$. Next we review the formula in (3). Let us decompose $f \in C_{d}^{n}(X)$ as $f=\sum_{0 \leq a \leq p-1} f_{a}\left(U_{1}, \ldots, U_{n-1}\right) \cdot T_{n}^{a}$, where we denote 
the $n$-th variable by $T_{n}$ instead of $U_{n}$. By an easy calculation, we have the following fundamental formula (see [M2, Lemma 3.2]), which the reader should keep in mind:

$$
\begin{aligned}
& \delta_{n}(f)\left(U_{1}, \ldots, U_{n}, T_{n+1}\right)=\sum_{0 \leq a \leq p-1} \delta_{n-1}\left(f_{a}\right)\left(U_{1}, \ldots, U_{n}\right) \cdot T_{n+1}^{a} \\
& \quad+(-1)^{n-1} \sum_{0 \leq a \leq p-1} f_{a}\left(U_{1}, \ldots, U_{n-1}\right) \cdot\left(\omega^{d} \cdot\left(U_{n}+\omega^{-1} T_{n+1}\right)^{a}-\left(U_{n}+T_{n+1}\right)^{a}\right) .
\end{aligned}
$$

By the following lemma shown in M1, M2, we may consider only the case of $\omega^{d}=1$.

Lemma 3.1 (피, Lemma 3.1]). If $\omega^{d} \neq 1$, then the complex $C_{d}^{n}(X)$ is acyclic.

Proof. Let $f \in C_{d}^{n}(X)$ be an $n$-cocycle. Applying $T_{n+1}=0$ to (3), we can form

$$
0=\delta_{n-1}\left(f_{0}\right)\left(U_{1}, \ldots, U_{n}\right)+(-1)^{n-1}\left(\omega^{d}-1\right) \sum_{0 \leq a \leq p-1} f_{a}\left(U_{1}, \ldots, U_{n-1}\right) \cdot U_{n}^{a} .
$$

We thus have $f=(-1)^{n}\left(\omega^{d}-1\right)^{-1} \cdot \delta_{n-1}\left(f_{0}\right)$, which implies that $f$ is cohomologous to zero.

Next, we consider the following submodule of $C^{n}(X)$ : for $n \geq 1$

$$
C^{n(1)}(X):=\left\{f_{0}\left(U_{1}, \ldots, U_{n-1}\right) \in C^{n}(X) \mid f_{0} \in \mathbb{Z}_{p}\left[U_{1}, \ldots, U_{n-1}\right]\right\},
$$

and $C^{0(1)}(X):=\{0\}$. Put $C_{d}^{n(1)}(X):=C^{n(1)}(X) \cap C_{d}^{n}(X)$. Since $\delta_{n}\left(C_{d}^{n(1)}(X)\right)$ is contained in $C_{d}^{n+1(1)}(X)$, we define the cohomologies as follows:

$$
\begin{gathered}
Z_{d}^{n(1)}(X):=\operatorname{Ker}\left(\delta_{n}\right), \\
B_{d}^{n(1)}(X):=\delta_{n-1}\left(C_{d}^{n-1(1)}(X)\right), \\
H_{d}^{n(1)}(X):=Z_{d}^{n(1)}(X) / B_{d}^{n(1)}(X) .
\end{gathered}
$$

We further define a cohomology of the quotient complex $C_{d}^{n}(X) / C_{d}^{n(1)}(X)$ as usual, i.e.,

$$
\begin{gathered}
Z_{d}^{n}(X):=\operatorname{Ker}\left(\delta_{n}\right) \cap C_{d}^{n}(X), \\
B_{d}^{n}(X):=\delta_{n-1}\left(C_{d}^{n-1}(X)\right), \\
H_{d}^{n(0)}(X):=Z_{d}^{n}(X) /\left(B_{d}^{n}(X)+Z_{d}^{n(1)}(X)\right) .
\end{gathered}
$$

These notation will be used later. Further, we denote $\bigoplus_{d} H_{d}^{n(1)}(X)$ and $\bigoplus_{d} H_{d}^{n(0)}(X)$ by $H^{n(1)}(X)$ and $H^{n(0)}(X)$, respectively. By Lemma 3.1, we have

$$
H^{n(0)} \cong \bigoplus_{d: \omega^{d}=1} H_{d}^{n(0)}(X), \quad H^{n(1)} \cong \bigoplus_{d: \omega^{d}=1} H_{d}^{n(1)}(X) .
$$

Further $c_{n}^{(1)}$ and $c_{n}^{(0)}$ denote $\operatorname{dim}\left(H^{n(1)}(X)\right)$ and $\operatorname{dim}\left(H^{n(0)}(X)\right)$, respectively. Note that by definition $c_{0}^{(0)}=c_{1}^{(1)}=1$ is clear. 
3.2. Quandle cohomology and a decomposition of quandle complex. In this subsection, we state a decomposition of and the dimension of the quandle cohomology group for an Alexander quandle of order $p$. We first show that the following short exact sequence splits:

$$
0 \longrightarrow C_{d}^{n(1)}(X) \stackrel{i_{d}^{n}}{\longrightarrow} C_{d}^{n}(X) \longrightarrow C_{d}^{n}(X) / C_{d}^{n(1)}(X) \longrightarrow 0 .
$$

Proposition 3.2 (cf. [M2, Theorem 3.12]). Let $X$ be an Alexander quandle of order $p$ with $\omega$. Let $s_{d}^{n}$ be the canonical projection $C_{d}^{n}(X) \rightarrow C_{d}^{n(1)}(X)$ as a linear map. Let $n \geq 2$.

(I) If $\omega^{d}=1$, then $s_{d}^{n}$ is a chain map. In particular, $H_{d}^{n}(X) \cong H_{d}^{n(0)}(X) \oplus$ $H_{d}^{n(1)}(X)$.

(II) If $\omega^{d}=1$, then the canonical inclusion $C_{d}^{n(1)}(X) \hookrightarrow C_{d}^{n-1}(X)$ induces an isomorphism $H_{d}^{n-1(0)}(X) \cong H_{d}^{n(1)}(X)$. As a result, $H^{n}(X) \cong H^{n-1(0)}(X) \oplus$ $H^{n(0)}(X)$.

This will be shown in the next section. In conclusion, for the study on $H^{n}(X)$, it is enough to search $H^{n(0)}(X)$. Furthermore, the following theorem determines $H^{n(0)}(X)$.

Theorem 3.3. Let $X$ be as above, and let $e$ be the order of $\omega$, and $c_{n}^{(0)}:=$ $\operatorname{dim}\left(H^{n(0)}(X)\right)$. Then

(I) $c_{0}^{(0)}=c_{2 e-1}^{(0)}=1$, and $c_{n}^{(0)}=0$ for $1 \leq n \leq 2 e-2$.

(II) $c_{n}^{(0)}=c_{n-2 e+2}^{(0)}+c_{n-2 e+1}^{(0)}+c_{n-2 e}^{(0)}$ for $n \geq 2 e$.

Our purpose in Sections 4 to 6 is to show this theorem, which is the key to proving Theorem 2.2 as mentioned in Subsection 2.3

Remark 3.4. Consequently, we can determine $H^{n}(X)$ as follows. Put the generating function $F_{c}(x):=\sum_{i>0} \operatorname{dim}\left(H^{i}(X)\right) x^{i} \in \mathbb{Z}[[x]]$. Since Proposition 3.2 follows $\operatorname{dim}\left(H^{n}(X)\right)=c_{n-1}^{(0)}+c_{n}^{(0)}$, Theorem 3.3 indicates $F_{c}(x)=\left(1-x^{2 e-2}+x^{2 e}\right)$. $\left(1-x^{2 e-2}-x^{2 e-1}-x^{2 e}\right)^{-1}$.

Furthermore, in Section 5.4 we will give concrete presentations of all $n$-cocycles which span $H^{n(0)}(X)$ (Corollary 5.8). Therefore, we can determine $\operatorname{dim}\left(H_{d}^{n}(X)\right)$, and $\operatorname{dim}\left(H_{d}^{n(0)}(X)\right)$ for any $d$ and $n$, although we omit the explicit formulae.

3.3. Calculus on quandle cochain groups. We will set up a differential operation and an integral operation on the quandle cochain group. Using them, we will show Proposition 3.2 at the end of this subsection.

We first define a homomorphism $\mathcal{D}_{d}^{n}: C_{d}^{n}(X) \rightarrow C_{d-1}^{n}(X)$ of degree -1 by

$$
\mathcal{D}_{d}^{n}\left(\sum_{0 \leq a \leq p-1} f_{a}\left(U_{1}, \ldots, U_{n-1}\right) \cdot T_{n}^{a}\right)=\sum_{0 \leq a \leq p-1} a \cdot f_{a}\left(U_{1}, \ldots, U_{n-1}\right) \cdot T_{n}^{a-1} .
$$

Note that $\operatorname{Ker}\left(\mathcal{D}_{d}^{n}\right)=C_{d}^{n(1)}(X)$ and that any element of the form $f_{p-1}\left(U_{1}, \ldots, U_{n-1}\right)$. $T_{n}^{p-1} \in C_{d-1}^{n}(X)$ is not contained in the image of $\mathcal{D}_{d}^{n}$. Further $\mathcal{D}_{d}^{n}$ is shown to be a chain homomorphism, that is, $\delta_{n} \circ \mathcal{D}_{d}^{n}=\mathcal{D}_{d}^{n+1} \circ \delta_{n}$.

On the other hand, for $n, d \in \mathbb{Z}$, we will introduce an integral operation from $\operatorname{Im}\left(\mathcal{D}_{d}^{n}\right)$ to $C_{d}^{n}(X)$. Let $f=\sum_{0 \leq a \leq p-2} f_{a}\left(U_{1}, \ldots, U_{n-1}\right) \cdot U_{n}^{a} \in \operatorname{Im}\left(\mathcal{D}_{d}^{n}\right)$. We then 
define the integration by the formula

$$
\int_{n}(f)\left(U_{1}, \ldots, U_{n}\right)=\sum_{0 \leq a \leq p-2}(a+1)^{-1} \cdot f_{a}\left(U_{1}, \ldots, U_{n-1}\right) \cdot U_{n}^{a+1} .
$$

Note that for $g=\sum_{0 \leq a \leq p-1} g_{a}\left(U_{1}, \ldots, U_{n-1}\right) \cdot U_{n}^{a} \in C_{d}^{n}(X),\left(\int_{n} \circ \mathcal{D}_{d}^{n}\right)(g)=$ $g-g_{0}$. Furthermore, by direct calculation we can show the following relation between the integration and $\delta_{n}$.

Lemma 3.5. For $f \in \operatorname{Im}\left(\mathcal{D}_{d}^{n-1}\right)$, let us regard $\int_{n-1}(f)$ as an element of $C_{d}^{n(1)}(X)$. Then

$$
\left(\delta_{n-1} \circ \int_{n-1}\right)(f)=\left(\int_{n} \circ \delta_{n-1}\right)(f)+(-1)^{n-1}\left(1-\omega^{d}\right) \int_{n-1}(f) \in C_{d}^{n}(X) .
$$

In particular, if $\omega^{d}=1$, then the integration commutes with the coboundary map $\delta_{n}$.

Proof of Proposition 3.2. To prove the part (I), we formulate any element $f \in$ $C_{d}^{n}(X)$ by $f=\sum_{k \leq p-1} f_{k}\left(U_{1}, \ldots, U_{n-1}\right) U_{n}^{k}$. Notice that the section $s_{d}^{n}: C_{d}^{n}(X) \rightarrow$ $C_{d}^{n(1)}(X)$ is defined by $s_{d}^{n}(f)=f_{0}\left(U_{1}, \ldots, U_{n-1}\right)$. We first note that $\left(\int_{n} \circ \mathcal{D}_{d}^{n}\right)(f-$ $\left.f_{0}\right)=f-f_{0} \in C_{d}^{n}(X)$ and that $s_{d}^{n} \circ \int_{n} \circ \mathcal{D}_{d}^{n}$ is the zero map by definitions. Hence,

$$
\begin{aligned}
s_{d}^{n+1}\left(\delta_{n}(f)\right) & =s_{d}^{n+1}\left(\delta_{n}\left(f-f_{0}\right)\right)+s_{d}^{n+1}\left(\delta_{n}\left(f_{0}\right)\right) \\
& =s_{d}^{n+1}\left(\delta_{n}\left(\left(\int_{n} \circ \mathcal{D}_{d}^{n}\right)\left(f-f_{0}\right)\right)\right)+\delta_{n}\left(f_{0}\right) \\
& =\left(s_{d}^{n+1} \circ \int_{n}\right)\left(\left(\delta_{n} \circ \mathcal{D}_{d}^{n}\right)\left(f-f_{0}\right)\right)+\delta_{n}\left(s_{d}^{n}(f)\right)=\delta_{n}\left(s_{d}^{n}(f)\right),
\end{aligned}
$$

where we use (6) in the third equality. Namely, the section $s_{d}^{n}$ is a chain map as desired.

To prove (II), reverify the canonical inclusion $\iota: C_{d}^{n(1)}(X) \rightarrow C_{d}^{n-1}(X)$ from the definitions of these complexes. This map induces a bijection between $C_{d}^{n(1)}(X)$ and $C_{d}^{n-1}(X) / C_{d}^{n-1(1)}(X)$. When $\omega^{d}=1$, formula (3) immediately concludes $\delta_{n}\left(f_{0}\right)=$ $\delta_{n-1}\left(\iota\left(f_{0}\right)\right)$. Thus, the bijection is also a chain isomorphism, which immediately leads to $H_{d}^{n(1)}(X) \cong H_{d}^{n-1(0)}(X)$ as required.

3.4. Mochizuki's techniques in the general case of $n$. In M1, M2, T. Mochizuki discovered all 2- and 3-cocycles of the quandle cohomology of a certain Alexander quandle. He reduced them to a quotient vector space in [M2, Section 3.2.4]. Following his techniques, in the general case of $n$, we will construct an isomorphism from $H_{d}^{n(0)}(X)$ to a quotient space given by (8) below. Assume that $f \in C_{d}^{n}(X)$ is an $n$-cocycle and $\omega^{d}=1$. Then by (3) we obtain

$$
\begin{gathered}
0=\sum_{0 \leq a \leq p-1} \delta_{n-1}\left(f_{a}\right)\left(U_{1}, \ldots, U_{n}\right) \cdot T_{n+1}^{a}-(-1)^{n} f_{a}\left(U_{1}, \ldots, U_{n-1}\right) \\
\cdot\left(\left(U_{n}+\omega^{-1} T_{n+1}\right)^{a}-\left(U_{n}+T_{n+1}\right)^{a}\right) .
\end{gathered}
$$

By comparing with the coefficients of $T_{n+1}^{1}$ on both the left and right sides, we have

$$
\delta_{n-1}\left(f_{1}\right)\left(U_{1}, \ldots, U_{n}\right)=(-1)^{n}\left(\omega^{-1}-1\right) \sum_{0 \leq a \leq p-1} f_{a}\left(U_{1}, \ldots, U_{n-1}\right) \cdot a \cdot U_{n}^{a-1} .
$$


Namely, $\delta_{n-1}\left(f_{1}\right)=(-1)^{n}\left(\omega^{-1}-1\right) \cdot \mathcal{D}_{d}^{n}(f)$. Hence $f_{1} \in \delta_{n-1}^{-1}\left(\operatorname{Im}\left(\mathcal{D}_{d}^{n}\right)\right)$. We thus obtain a homomorphism $\phi: Z_{d}^{n}(X) \rightarrow \delta_{n-1}^{-1}\left(\operatorname{Im}\left(\mathcal{D}_{d}^{n}\right)\right)$ given by $\phi(f)=f_{1}$.

Lemma 3.6. Let $g=\sum_{a} g_{a}\left(U_{1}, \ldots, U_{n-2}\right) \cdot T_{n-1}^{a} \in C_{d}^{n-1}(X)$. If $\omega^{d}=1$, then

$$
\phi\left(\delta_{n-1}(g)\right)=\delta_{n-2}\left(g_{1}\right)+(-1)^{n}\left(\omega^{-1}-1\right) \cdot \mathcal{D}_{d}^{n-1}(g) .
$$

Proof. Straightforward.

Further, it is evident that $\phi\left(Z_{d}^{n(1)}(X)\right)=\{0\}$. Hence the homomorphism $\phi$ induces

$$
\bar{\phi}: \frac{\mathrm{Z}_{d}^{n}(X)}{B_{d}^{n}(X)+\mathrm{Z}_{d}^{n(1)}(X)} \longrightarrow \frac{\delta_{n-1}^{-1}\left(\operatorname{Im}\left(\mathcal{D}_{d}^{n}\right)\right)}{\operatorname{Im}\left(\mathcal{D}_{d}^{n-1}\right)+B_{d-1}^{n-1}(X)} .
$$

T. Mochizuki showed that the homomorphism $\bar{\phi}$ is an isomorphism in the case of $n=3$ [M2, Lemma 3.17]. It holds for general $n$ as follows:

Proposition 3.7. If $\omega^{d}=1$, then the homomorphism $\bar{\phi}$ is an isomorphism. Furthermore, for a representative of the form $g_{p-1}\left(U_{1}, \ldots, U_{n-2}\right) \cdot U_{n-1}^{p-1} \in \delta_{n-1}^{-1}\left(\operatorname{Im}\left(\mathcal{D}_{d}^{n}\right)\right)$, the preimage via $\bar{\phi}$ is represented by a polynomial $\hat{g}$ of the form

$$
\begin{aligned}
\hat{g}\left(U_{1}, \ldots, T_{n}\right)= & g_{p-1}\left(U_{1}, \ldots, U_{n-2}\right) \\
& \cdot \frac{\left(1-\omega^{-1}\right) T_{n}^{p}-\left(U_{n-1}+T_{n}\right)^{p}+\left(U_{n-1}+\omega^{-1} T_{n}\right)^{p}}{\left(1-\omega^{-1}\right) p} .
\end{aligned}
$$

Proof. We first show that the homomorphism $\bar{\phi}$ is injective. Let $f \in C_{d}^{n}(X)$ be an $n$-cocycle such that $\bar{\phi}(f)=0$. Then we have $f_{1}=\delta_{n-2}(h)+\mathcal{D}_{d}^{n-1}(g)$ for some $h \in C_{d-1}^{n-2}(X)$ and $g \in C_{d}^{n-1}(X)$. Put an $n$-cocycle $\bar{f}$ given by $\bar{f}=f+$ $(-1)^{n}\left(1-\omega^{-1}\right)^{-1} \cdot \delta_{n-1}(g) \in C_{d}^{n}(X)$. It follows from (7) that $\mathcal{D}_{d}^{n}(\bar{f})=\mathcal{D}_{d}^{n}(f)+$ $(-1)^{n}\left(1-\omega^{-1}\right)^{-1} \cdot \delta_{n-1}\left(\mathcal{D}_{d}^{n-1}(g)\right)=0$. Since $\operatorname{Ker}\left(\mathcal{D}_{d}^{n}\right)=C_{d}^{n(1)}(X)$, it implies $f \in B_{d}^{n}(X)+Z_{d}^{n(1)}(X)$.

Next, we will show its surjectivity. For $g \in C_{d-1}^{n-1}(X)$ satisfying $\delta_{n-1}(g) \in$ $\operatorname{Im}\left(\mathcal{D}_{d}^{n}\right)$, we shall construct an $n$-cocycle $\hat{g}$ so that $\phi(\hat{g})=g$. Since we consider $g$ modulo $\operatorname{Im}\left(\mathcal{D}_{d}^{n-1}\right)$, we may deal with only $g$ of the form

$$
g\left(U_{1}, \ldots, U_{n-2}, T_{n-1}\right)=g_{p-1}\left(U_{1}, \ldots, U_{n-2}\right) \cdot T_{n-1}^{p-1},
$$

where $g_{p-1} \in C_{d-p}^{n-2}(X)$. Then we define a polynomial $\hat{g}$ by

$$
\hat{g}\left(U_{1}, \ldots, U_{n-1}, T_{n}\right)=\int_{n}\left(\delta_{n-1}\left(g_{p-1}\left(U_{1}, \ldots, U_{n-2}\right) \cdot U_{n-1}^{p-1}\right)\right) \in C_{d}^{n}(X) .
$$

We now check that $\hat{g}$ is an $n$-cocycle. Actually, since $\omega^{d}=1$, equality (6) leads to

$$
\delta_{n}(\hat{g})=\int_{n+1}\left(\delta_{n} \circ \delta_{n-1}\left(g_{p-1}\left(U_{1}, \ldots, U_{n-2}\right) \cdot U_{n-1}^{p-1}\right)\right)=0 .
$$

It remains to show that $\phi(\hat{g})=g$ and (9). For this, Lemma 3.9 below immediately indicates

$$
\delta_{n-2}\left(g_{p-1}\right)\left(U_{1}, \ldots, U_{n-1}\right)=(-1)^{n}\left(1-\omega^{-1}\right) \cdot g_{p-1}\left(U_{1}, \ldots, U_{n-2}\right) \in C_{d-p}^{n-1(1)}(X) .
$$

Using this equality, let us expand the right side in (10). Then we obtain the required formula (9). Further, by the formula (9), we easily see $\phi(\hat{g})=g$. 
Remark 3.8. We have easily obtained the simple formula (9) above. However, T. Mochizuki originally showed a slightly complicated formula to produce $n$-cocycles of Alexander quandles on $\mathbb{R}$ (see [M1, Proposition 3.1] for details). By pulling back the formula to $\mathbb{Z}_{p}$, he found a non-trivial 3-cocycle of the dihedral quandle of order $p$ [M1, Theorem 3.1].

The following lemma will be used several times later on.

Lemma 3.9. Let $g \in C_{d-1}^{n-1}(X)$ with no term of $C_{d-1}^{n-1(1)}(X)$. Let us decompose $g$ as $g=\sum_{1 \leq a \leq p-1} g_{a}\left(U_{1}, \ldots, U_{n-2}\right) \cdot U_{n-1}^{a}$. If $\delta_{n-1}(g) \in \operatorname{Im}\left(\mathcal{D}_{d}^{n}\right)$, then it satisfies

$$
\delta_{n-2}\left(g_{p-1}\right)\left(U_{1}, \ldots, U_{n-1}\right)=(-1)^{n}\left(1-\omega^{d-1}\right) \cdot g_{p-1}\left(U_{1}, \ldots, U_{n-2}\right) \in C_{d-p}^{n-1(1)}(X) .
$$

Proof. By (3) we thus have

$$
\begin{aligned}
& \delta_{n-1}(g)\left(U_{1}, \ldots, U_{n-1}, T_{n}\right)=\sum_{1 \leq a \leq p-1} \delta_{n-2}\left(g_{a}\right)\left(U_{1}, \ldots, U_{n-1}\right) \cdot T_{n}^{a} \\
& +(-1)^{n} g_{a}\left(U_{1}, \ldots, U_{n-2}\right)\left(\omega^{d-1} \cdot\left(U_{n-1}+\omega^{-1} T_{n}\right)^{a}-\left(U_{n-1}+T_{n}\right)^{a}\right) .
\end{aligned}
$$

Since $\delta_{n-1}(g) \in \operatorname{Im}\left(\mathcal{D}_{d}^{n}\right)$, by comparing with the coefficients of $T_{n}^{p-1}$ on the right side, we obtain equality (12).

Before going into the next section, we show Proposition 3.10 on the forms of the cocycles. For this, we now introduce the following polynomial $E_{n-1}^{\omega} \in \mathbb{Z}_{p}\left[U_{n-1}, U_{n}\right]$ :

$$
\begin{aligned}
E_{n-1}^{\omega}\left(U_{n-1}, U_{n}\right) & =\left(\left(U_{n-1}+U_{n}\right)^{p}-\left(1-\omega^{-1}\right) U_{n}^{p}-\left(U_{n-1}+\omega^{-1} U_{n}\right)^{p}\right) / p \\
& \equiv \sum_{1 \leq j \leq p-2}(-1)^{j-1} j^{-1} \cdot\left(1-\omega^{-j}\right) \cdot U_{n-1}^{p-j} \cdot U_{n}^{j}(\bmod p) .
\end{aligned}
$$

Proposition 3.10. Let $X$ be an Alexander quandle of order $p$. Then $H^{n(0)}(X)$ is generated by some elements of the forms $g_{p-1} \cdot E_{n-1}^{\omega}$, where $g_{p-1}$ is contained in $C_{d-p}^{n-2}(X)$ for some $d$ and satisfies $\delta_{n-2}\left(g_{p-1}\right)=(-1)^{n}\left(1-\omega^{-1}\right) \cdot g_{p-1} \in C_{d-p}^{n-1(1)}(X)$ and $\omega^{d}=1$.

Proof. It follows from Proposition 3.7 that $n$-cocycles of the forms $g_{p-1} \cdot E_{n-1}^{\omega}$ generate $H^{n(0)}(X)$. Moreover, by (11) in the previous proof, $g_{p-1}$ have to satisfy $\delta_{n-2}\left(g_{p-1}\right)=(-1)^{n}\left(1-\omega^{-1}\right) \cdot g_{p-1} \in C_{d-p}^{n-1(1)}(X)$ and $\omega^{d}=1$.

\section{Proof of Theorem 3.3(I)}

Throughout this section we fix the order $e$ of $\omega$, in other words, $e$ is the minimal number of satisfying $\omega^{e}=1$. Also, we assume that $\omega^{d}=1$. In Section 4.1, we will search the quotient vector space in (8) in the case $\omega \neq-1$. We will construct a homomorphism $\bar{\Theta}_{i}$ and show that the map is an isomorphism (Proposition 4.2). Roughly speaking, the isomorphism makes the degree of the quotient space a lower degree. In Section 4.3, as a corollary, we will prove Theorem 3.3. (I), i.e., $c_{1}^{(0)}=\cdots=c_{2 e-2}^{(0)}=0$ and $c_{0}^{(0)}=c_{2 e-1}^{(0)}=1$. 
4.1. Isomorphisms $\bar{\Theta}_{i}$. We will construct a map given below in (15). In this subsection we assume that $\omega \neq-1$, and we fix $1 \leq i \leq e-2$ such that $n \geq$ $2 i+2$. To begin with, we put $g=\sum_{j} g_{j}\left(U_{1}, \ldots, U_{n-2 i}\right) \cdot U_{n-2 i+1}^{j}$ contained in $\delta_{n-2 i+1}^{-1}\left(\operatorname{Im}\left(\mathcal{D}_{d-i p+p}^{n-2 i+2}\right)\right)$. By Lemma 3.9 we have

$$
\delta_{n-2 i}\left(g_{p-1}\right)=(-1)^{n-2 i+2}\left(1-\omega^{d-i p+p-1}\right) \cdot g_{p-1}=(-1)^{n}\left(1-\omega^{-i}\right) \cdot g_{p-1} .
$$

Moreover, we decompose

$$
g_{p-1}\left(U_{1}, \ldots, U_{n-2 i+1}\right)=\sum_{1 \leq k \leq p-1} g_{k, p-1}\left(U_{1}, \ldots, U_{n-2 i}\right) \cdot U_{n-2 i+1}^{k} .
$$

Then the above equalities mean

$$
\begin{aligned}
(-1)^{n} & \left(1-\omega^{-i}\right) \cdot g_{p-1}\left(U_{1}, \ldots, U_{n-2 i}\right) \\
= & \sum_{1 \leq k \leq p-1} \delta_{n-2 i-1}\left(g_{k, p-1}\left(U_{1}, \ldots, U_{n-2 i}\right) \cdot T_{n-2 i+1}^{k}\right. \\
& \quad+(-1)^{n-1} \sum_{1 \leq k \leq p-1} g_{k, p-1}\left(U_{1}, \ldots, U_{n-2 i-1}\right) \\
& \cdot\left(\omega^{d-i p}\left(U_{n-2 i}+\omega^{-1} T_{n-2 i+1}\right)^{k}-\left(U_{n-2 i}-T_{n-2 i+1}\right)^{k}\right) .
\end{aligned}
$$

Comparing the coefficients of $T_{n-2 i+1}^{1}$ in both the left and right sides shows an equality

$$
\delta_{n-2 i-1}\left(g_{1, p-1}\right)=(-1)^{n}\left(\omega^{-i-1}-1\right) \cdot \mathcal{D}_{d-i p}^{n-2 i}\left(g_{p-1}\right) .
$$

Therefore we obtain a homomorphism

$$
\Theta_{i}: \delta_{n-2 i+1}^{-1}\left(\operatorname{Im}\left(\mathcal{D}_{d-i p+p}^{n-2 i+2}\right)\right) \longrightarrow \delta_{n-2 i-1}^{-1}\left(\operatorname{Im}\left(\mathcal{D}_{d-i p}^{n-2 i}\right)\right),
$$

given by $\Theta_{i}(g)=g_{1, p-1}$. We can verify the following lemma, similar to Lemma 3.6 .

Lemma 4.1. Let $h$ be an element of $C_{d-i p+p-1}^{n-2 i}(X)$. We decompose

$$
h=\sum_{a, b} h_{a, b}\left(U_{1}, \ldots, U_{n-2 i-2}\right) \cdot U_{n-2 i-1}^{a} \cdot T_{n-2 i}^{b} .
$$

If $\omega^{d}=1$, then

$$
\begin{aligned}
\Theta_{i}\left(\delta_{n-2 i}(h)\right)= & \delta_{n-2 i-2}\left(h_{1, p-1}\right)+(-1)^{n}\left(\omega^{-i-1}-1\right) \\
& \cdot\left(\sum_{1 \leq a \leq p-1} a \cdot h_{a, p-1}\left(U_{1}, \ldots, U_{n-2 i-2}\right) \cdot U_{n-2 i-1}^{a-1}\right) .
\end{aligned}
$$

Further, it is clear that $\Theta_{i}\left(\operatorname{Im}\left(\mathcal{D}_{d-i p+p}^{n-2 i+1}\right)\right)=\{0\}$. Hence, the homomorphism $\Theta_{i}$ induces

$$
\bar{\Theta}_{i}: \frac{\delta_{n-2 i+1}^{-1}\left(\operatorname{Im}\left(\mathcal{D}_{d-i p+p}^{n-2 i+2}\right)\right)}{\operatorname{Im}\left(\mathcal{D}_{d-i p+p}^{n-2 i+1}\right)+B_{d-i p+p-1}^{n-2 i+1}(X)} \longrightarrow \frac{\delta_{n-2 i-1}^{-1}\left(\operatorname{Im}\left(\mathcal{D}_{d-i p}^{n-2 i}\right)\right)}{\operatorname{Im}\left(\mathcal{D}_{d-i p}^{n-2 i-1}\right)+B_{d-i p-1}^{n-2 i-1}(X)} .
$$

We remark that if $i=1$, then the left side is exactly the right side in (8).

In order to state Proposition 4.2, we now introduce the polynomial

$$
E_{n-2 i-1}^{\omega, i}\left(U_{n-2 i-1}, U_{n-2 i}\right) \in \mathbb{Z}_{p}\left[U_{n-2 i-1}, U_{n-2 i}\right]
$$


given by the formula

$$
\begin{aligned}
\left(\left(1-\omega^{-i-1}\right) U_{n-2 i}^{p}+\omega^{-i}\left(U_{n-2 i-1}+\omega^{-1} U_{n-2 i}\right)^{p}\right. & \\
& \left.-\left(U_{n-2 i-1}+U_{n-2 i}\right)^{p}+\left(1-\omega^{-i}\right) U_{n-2 i-1}^{p}\right) / p \\
\equiv & \sum_{1 \leq j \leq p-1}(-1)^{j-1} j^{-1} \cdot\left(\omega^{-i-j}-1\right) \cdot U_{n-2 i-1}^{p-j} \cdot U_{n-2 i}^{j}(\bmod p) .
\end{aligned}
$$

Proposition 4.2. If $\omega^{d}=1, n-2 i-2 \geq 0$, and $0<i \leq e-2$, then $\bar{\Theta}_{i}$ is an isomorphism. Further, for a representative of the form $g_{p-1}^{\prime}\left(U_{1}, \ldots, U_{n-2 i-2}\right)$. $U_{n-2 i-1}^{p-1} \in \delta_{n-2 i-1}^{-1}\left(\operatorname{Im}\left(\mathcal{D}_{d-i p}^{n-2 i}\right)\right)$, the element $\left(\bar{\Theta}_{i}\right)^{-1}\left(g_{p-1}^{\prime} \cdot U_{n-2 i-1}^{p-1}\right)$ is represented by the formula

$$
(-1)^{n}\left(\omega^{-i-1}-1\right)^{-1} \cdot g_{p-1}^{\prime}\left(U_{1}, \ldots, U_{n-2 i-2}\right) \cdot E_{n-2 i-1}^{\omega, i}\left(U_{n-2 i-1}, U_{n-2 i}\right) \cdot T_{n-2 i+1}^{p-1} \text {. }
$$

Before going into the proof, we make some remarks. Since $\omega^{d}=1$, we have $\omega^{d-i p-p-1}=\omega^{-i}$. Also, note that $1-\omega^{-i}, 1-\omega^{-i-1} \in \mathbb{Z}_{p}$ are non-zero from the definition of $e$.

Proof. First, we study the injectivity. Assume that $\bar{\Theta}_{i}(g)=0$, that is, $g_{1, p-1} \in$ $\operatorname{Im}\left(\mathcal{D}_{d-i p}^{n-2 i-1}\right)+B_{d-i p-1}^{n-2 i-1}(X)$. We will show that such $g$ can be killed below. We have $g_{1, p-1}=\mathcal{D}_{d-i p}^{n-2 i-1}\left(g^{\prime}\right)+\delta_{n-2 i-2}(h)$ for some $g^{\prime} \in C_{d-i p}^{n-2 i-1}(X)$ and $h \in C_{d-i p-1}^{n-2 i-2}(X)$. We may assume that the coefficient of $U_{n-2 i-1}^{0}$ in $g^{\prime}$ is zero. Then we integrate (14) by $T_{n-2 i}$, and obtain

$$
\begin{aligned}
& (-1)^{n}\left(\omega^{-i-1}-1\right) \cdot g_{p-1}=\left(\int_{n-2 i} \circ \delta_{n-2 i-1} \circ \mathcal{D}_{d-i p}^{n-2 i-1}\right)\left(g^{\prime}\right) \\
& =\left(\int_{n-2 i} \circ \mathcal{D}_{d-i p}^{n-2 i}\right)\left(\delta_{n-2 i-1}\left(g^{\prime}\right)\right)=\delta_{n-2 i-1}\left(g^{\prime}\right)-(-1)^{n}\left(\omega^{-i}-1\right) \cdot g^{\prime}
\end{aligned}
$$

where the last equality is obtained from that the coefficient of $U_{n-2 i-1}^{0}$ in $\delta_{n-2 i-1}\left(g^{\prime}\right)$ is $(-1)^{n-2 i-2}\left(\omega^{d-i p}-1\right) \cdot g^{\prime}$ by (3). We put $\check{g}\left(U_{1}, \ldots, U_{n-2 i}\right):=g^{\prime}\left(U_{1}, \ldots, U_{n-2 i-1}\right)$. $U_{n-2 i}^{p-1} \in C_{d-i p+p-1}^{n-2 i}(X)$. Then we have

$$
\begin{aligned}
\delta_{n-2 i}(\check{g}) & \left(U_{1}, \ldots, U_{n-2 i}, T_{n-2 i+1}\right) \\
= & \delta_{n-2 i-1}\left(g^{\prime}\right)\left(U_{1}, \ldots, U_{n-2 i}\right) \cdot T_{n-2 i+1}^{p-1} \\
& +(-1)^{n+1} g^{\prime}\left(U_{1}, \ldots, U_{n-2 i-1}\right) \\
& \cdot\left(\omega^{-i}\left(U_{n-2 i}+\omega^{-1} T_{n-2 i+1}\right)^{p-1}-\left(U_{n-2 i}+T_{n-2 i+1}\right)^{p-1}\right) \\
\equiv & (-1)^{n}\left(\left(\omega^{-i-1}-1\right) \cdot g_{p-1}+\left(\omega^{-i}-1\right) \cdot g^{\prime}\right)\left(U_{1}, \ldots, U_{n-2 i}\right) \cdot T_{n-2 i+1}^{p-1} \\
& +(-1)^{n+1}\left(\omega^{-i}-1\right) \cdot g^{\prime}\left(U_{1}, \ldots, U_{n-2 i-1}\right) \cdot T_{n-2 i+1}^{p-1} \\
= & (-1)^{n}\left(\omega^{-i-1}-1\right) \cdot g_{p-1}\left(U_{1}, \ldots, U_{n-2 i}\right) \cdot T_{n-2 i+1}^{p-1} \\
\equiv & (-1)^{n}\left(\omega^{-i-1}-1\right) \cdot g\left(U_{1}, \ldots, U_{n-2 i}, T_{n-2 i+1}\right) \bmod \left(\operatorname{Im}\left(\mathcal{D}_{d-i p+p}^{n-2 i+1}\right)\right),
\end{aligned}
$$

where the second equality is derived from (18). We remark that $\omega^{i+1} \neq 1$, since $0<i<e-1$. The above equalities imply that $g \in C_{d-i p+p-1}^{n-2 i+1}(X)$ is killed by $\check{g}$ as required. 
Next, we will show the surjectivity. We put $g^{\prime} \in \delta_{n-2 i-1}^{-1}\left(\operatorname{Im}\left(\mathcal{D}_{d-i p}^{n-2 i}\right)\right)$. Considering it modulo $\operatorname{Im}\left(\mathcal{D}_{d-i p}^{n-2 i-1}\right)$, we may assume that $g^{\prime}$ is of the form

$$
g^{\prime}\left(U_{1}, \ldots, U_{n-2 i-1}\right)=g_{p-1}^{\prime}\left(U_{1}, \ldots, U_{n-2 i-2}\right) \cdot U_{n-2 i-1}^{p-1} .
$$

Here we claim that the following polynomial $\breve{g}^{\prime}$ satisfies $\delta_{n-2 i+1}\left(\breve{g}^{\prime}\right) \in \operatorname{Im}\left(\mathcal{D}_{d-i p+p}^{n-2 i+2}\right)$ :

$$
\breve{g}^{\prime}\left(U_{1}, \ldots, U_{n-2 i}, T_{n-2 i+1}\right):=\left(\int_{n-2 i} \circ \delta_{n-2 i-1}\right)\left(g^{\prime}\right)\left(U_{1}, \ldots, U_{n-2 i}\right) \cdot T_{n-2 i+1}^{p-1}
$$

Indeed, the coefficient of $T_{n-2 i+2}^{p-1}$ in $\delta_{n-2 i+1}\left(\breve{g}^{\prime}\right)$ vanishes, since $(-1)^{n} \delta_{n-2 i+1}\left(\breve{g}^{\prime}\right)$ is reduced to be

$$
\begin{aligned}
&(-1)^{n} \delta_{n-2 i+1}\left(\left(\left(\int_{n-2 i} \circ \delta_{n-2 i-1}\right)\left(g^{\prime}\right)\right) \cdot U_{n-2 i+1}^{p-1}\right) \\
&=(-1)^{n} \delta_{n-2 i}\left(\left(\int_{n-2 i} \circ \delta_{n-2 i-1}\right)\left(g^{\prime}\right)\right) \cdot T_{n-2 i+2}^{p-1}+\left(\left(\int_{n-2 i} \circ \delta_{n-2 i-1}\right)\left(g^{\prime}\right)\right) \\
& \cdot\left(\left(\omega^{d-i p+p-1}\left(U_{n-2 i+1}+\omega^{-1} T_{n-2 i+2}\right)^{p-1}-\left(U_{n-2 i+1}+T_{n-2 i+2}\right)^{p-1}\right)\right. \\
&=(-1)^{n}\left(\left(\int_{n-2 i+1} \circ \delta_{n-2 i} \circ \delta_{n-2 i-1}\right)\left(g^{\prime}\right)\right. \\
&\left.+(-1)^{n}\left(1-\omega^{d-i p+p-1}\right)\left(\int_{n-2 i}^{\circ} \circ \delta_{n-2 i-1}\right)\left(g^{\prime}\right)\right) \cdot T_{n-2 i+2}^{p-1}+\int_{n-2 i}\left(\delta_{n-2 i-1}\left(g^{\prime}\right)\right) \\
& \cdot\left(\omega^{-i}\left(\omega U_{n-2 i+1}+T_{n-2 i+2}\right)^{p-1}-\left(U_{n-2 i+1}+T_{n-2 i+2}\right)^{p-1}\right) \\
&= \int_{n-2 i}\left(\delta_{n-2 i-1}\left(g^{\prime}\right)\right) \cdot\left(\left(1-\omega^{-i}\right) T_{n-2 i+2}^{p-1}\right. \\
&\left.+\omega^{-i}\left(\omega U_{n-2 i+1}+T_{n-2 i+2}\right)^{p-1}-\left(U_{n-2 i+1}+T_{n-2 i+2}\right)^{p-1}\right)
\end{aligned}
$$

where the first and second equalities are obtained from (3) and (6), respectively.

Finally we will show that the above $(-1)^{n} \breve{g}^{\prime}$ is equal to (17), immediately leading to $\Theta_{i}\left(\breve{g}^{\prime}\right)=g^{\prime}$. For this, note that by Lemma 3.9 we obtain

$$
\delta_{n-2 i-2}\left(g_{p-1}^{\prime}\right)\left(U_{1}, \ldots, U_{n-2 i-1}\right) \cdot U_{n-2 i}^{p-1}=(-1)^{n}\left(1-\omega^{-i}\right) g_{p-1}^{\prime}\left(U_{1}, \ldots, U_{n-2 i-2}\right) .
$$

Therefore, by using (3), $(-1)^{n} \delta_{n-2 i-1}\left(g^{\prime}\right)\left(U_{1}, \ldots, U_{n-2 i}\right)$ equals

$$
\begin{aligned}
(-1)^{n} \delta_{n-2 i-2}\left(g_{p-1}^{\prime}\right)\left(U_{1}, \ldots, U_{n-2 i-1}\right) \cdot U_{n-2 i}^{p-1}+g_{p-1}^{\prime}\left(U_{1}, \ldots, U_{n-2 i-2}\right) \\
\\
\cdot\left(\omega^{-i}\left(U_{n-2 i-1}+\omega^{-1} U_{n-2 i}\right)^{p-1}-\left(U_{n-2 i-1}+U_{n-2 i}\right)^{p-1}\right) \\
=g_{p-1}^{\prime}\left(U_{1}, \ldots, U_{n-2 i-2}\right) \\
\quad \cdot\left(\left(1-\omega^{-i}\right) U_{n-2 i}^{p-1}+\omega^{-i}\left(U_{n-2 i-1}+\omega^{-1} U_{n-2 i}\right)^{p-1}-\left(U_{n-2 i-1}+U_{n-2 i}\right)^{p-1}\right) .
\end{aligned}
$$

Hence, by integrating this, $(-1)^{n} \breve{g}^{\prime}$ in (19) turns out to be the required formula in (17).

4.2. Composition of $\bar{\Theta}_{i}$ 's. We will consider the composition of $\bar{\Theta}_{i}$ 's in Corollary 4.3. For this, we prepare the following product of the polynomials $E_{n-2 i}^{\omega, i}$ : if $\omega \neq-1$, we define

$$
E_{n-2 e+3 \leq n-2}^{\omega}\left(U_{n-2 e+3}, \ldots, U_{n-2}\right):=\prod_{1 \leq i \leq e-2} E_{n-2 k-1}^{\omega, i}\left(U_{n-2 i-1}, U_{n-2 i}\right) .
$$


Further, when $\omega=-1$, we define $E_{n-2 e+3 \leq n-2}^{\omega}$ to be $1 \in \mathbb{Z}_{p}$. We then reach a conclusion on the composition of the isomorphisms presented in Proposition 4.2 as follows.

Corollary 4.3. If $n \geq 2 e-1$ and $\omega \neq-1$, then the composite map $\bar{\Theta}_{e-2} \circ \cdots \circ \bar{\Theta}_{1}$ gives rise to an isomorphism

$$
\frac{\delta_{n-1}^{-1}\left(\operatorname{Im}\left(\mathcal{D}_{d}^{n}\right)\right)}{\operatorname{Im}\left(\mathcal{D}_{d}^{n-1}\right)+B_{d}^{n-1}(X)} \longrightarrow \frac{\delta_{n-2 e+3}^{-1}\left(\operatorname{Im}\left(\mathcal{D}_{d-e p+2 p}^{n-2 e+4}\right)\right)}{\operatorname{Im}\left(\mathcal{D}_{d-e p+2 p}^{n-2 e+3}\right)+B_{d-e p+2 p-1}^{n-2 e+3}(X)} .
$$

Further, for a representative of the form

$$
g\left(U_{1}, \ldots, U_{n-2 e+2}\right) \cdot U_{n-2 e+3}^{p-1} \in \delta_{n-2 e+3}^{-1}\left(\operatorname{Im}\left(\mathcal{D}_{d-e p+2 p}^{n-2 e+4}\right)\right),
$$

the preimage $\left(\bar{\Theta}_{e-2} \circ \cdots \circ \bar{\Theta}_{1}\right)^{-1}\left(g \cdot U_{n-2 e+3}^{p-1}\right)$ is represented by

$$
g\left(U_{1}, \ldots, U_{n-2 e+2}\right) \cdot E_{n-2 e+3 \leq n-2}^{\omega}\left(U_{n-2 e+3}, \ldots, U_{n-2}\right) \cdot T_{n-1}^{p-1} .
$$

Proof. This is proven according to Proposition 4.2 and the presentation of (19).

In Section 5, we will deal with the right side of (21).

We now consider the composition of $\bar{\Theta}_{i}$ and $\bar{\phi}$, where $\bar{\phi}$ is given by (8), and we present $n$-cocycles. Note that the following statement also holds for $\omega=-1$.

Corollary 4.4. Assume $n \geq 2 e-1$ and $\omega^{d}=1$. Let $g \in C_{d-e p+p}^{n-2 e+2}(X)$ be divisible by $U_{n-2 e+2}$. If $\omega \neq 0,1$ and $\delta_{n-2 e+2}(g)=(-1)^{n}(1-\omega) \cdot g \in C_{d-e p+p}^{n-2 e+3(1)}(X)$, then $g\left(U_{1}, \ldots, U_{n-2 e+2}\right) \cdot U_{n-2 e+3}^{p-1}$ is contained in $\delta_{n-2 e+3}^{-1}\left(\operatorname{Im}\left(\mathcal{D}_{d-e p+2 p}^{n-2 e+4}\right)\right)$. Moreover, for such $g$, the following polynomial is an n-cocycle of degree $d$ :

$$
g\left(U_{1}, \ldots, U_{n-2 e+2}\right) \cdot E_{n-2 e+3 \leq n-2}^{\omega}\left(U_{n-2 e+3}, \ldots, U_{n-2}\right) \cdot E_{n-1}^{\omega}\left(U_{n-1}, U_{n}\right) .
$$

Proof. The former is obtained by direct calculation similar to the proof in Lemma 3.9 .

We will show the latter part. Note that if $\omega=-1$, i.e., $e=2$, then the above statement is the same as Proposition 3.7. For $\omega \neq-1$, since $g\left(U_{1}, \ldots, U_{n-2 e+2}\right)$. $U_{n-2 e+3}^{p-1} \in \delta_{n-2 e+3}^{-1}\left(\operatorname{Im}\left(\mathcal{D}_{d-e p+2 p}^{n-2 e+4}\right)\right)$, then Corollary 4.3 yields $g\left(U_{1}, \ldots, U_{n-2 e+2}\right)$. $E_{n-2 e+3 \leq n-2}^{\omega} \cdot T_{n-1}^{p-1} \in \delta_{n-1}^{-1}\left(\operatorname{Im}\left(\mathcal{D}_{d-1}^{n}\right)\right)$. Moreover, according to Proposition 3.7 we conclude that the polynomial presented by (22) is an $n$-cocycle.

\subsection{The proof of Theorem $[\mathbf{3 . 3}(\mathrm{I})$.}

Proof. First, the proof in the cases $n \leq 3$ or $\omega=-1$ immediately results from the following facts. As mentioned in Section 3.1, $c_{0}^{(0)}=1$. Further, we easily check $Z^{1(0)}(X)=0$, which implies $c_{1}^{(0)}=0$. For $n=2, H_{Q}^{2}\left(X ; \mathbb{Z}_{p}\right) \cong 0$ is shown [M1, Corollary 2.2]. It is known [M1, Theorem 3.1] that $H^{3(0)}(X) \cong \mathbb{Z}_{p}$ if $\omega=-1$, and that $H^{3(0)}(X) \cong 0$ if $\omega \neq-1$.

Next, we may assume that $n \geq 4$ and $\omega \neq 1,0,-1$. For the proof of $c_{1}^{(0)}=\cdots=$ $c_{2 e-2}^{(0)}=0$, by Proposition 3.7 it suffices to show that the right side of (8) vanishes for $n<2 e-1$. For this, we consider the two cases where $n$ is odd or even.

First we consider the case where $n$ is even. Proposition 4.2 ensures an isomorphism

$$
\frac{\delta_{n-1}^{-1}\left(\operatorname{Im}\left(\mathcal{D}_{d}^{n}\right)\right)}{\operatorname{Im}\left(\mathcal{D}_{d}^{n-1}\right)+B_{d-1}^{n-1}(X)} \stackrel{\simeq}{\longrightarrow} \frac{\delta_{1}^{-1}\left(\operatorname{Im}\left(\mathcal{D}_{d+p-n p / 2}^{2}\right)\right)}{\operatorname{Im}\left(\mathcal{D}_{d+p-n p / 2}^{1}\right)+B_{d-1+p-n p / 2}^{1}(X)},
$$


given by the composite $\bar{\Theta}_{n / 2-1} \circ \cdots \circ \bar{\Theta}_{1}$. Any element of the right side is represented by $a_{1} \cdot U_{1}^{p-1}$ for some $a_{1} \in \mathbb{Z}_{p}$. Assume $a_{1} \neq 0$. By the homogenous degree we verify $p-1=d+p-n p / 2-1$, i.e., $d=p n / 2$. Since $n / 2 \leq e-1$ by assumption, we have $\omega^{d}=\omega^{n / 2} \neq 1$, which contradicts $\omega^{d}=1$. Hence $a_{1}=0$, that is, the above right side has no non-trivial elements. Therefore, $H^{n(0)}(X) \cong 0$.

On the other hand, in the odd case of $n$, Proposition 4.2 gives rise to an isomorphism

$$
\frac{\delta_{n-1}^{-1}\left(\operatorname{Im}\left(\mathcal{D}_{d}^{n}\right)\right)}{\operatorname{Im}\left(\mathcal{D}_{d}^{n-1}\right)+B_{d-1}^{n-1}(X)} \stackrel{\simeq}{\longrightarrow} \frac{\delta_{2}^{-1}\left(\operatorname{Im}\left(\mathcal{D}_{d-p(n-3) / 2}^{3}\right)\right)}{\operatorname{Im}\left(\mathcal{D}_{d-p(n-3) / 2}^{2}\right)+B_{d-1-p(n-3) / 2}^{2}(X)},
$$

obtained from the composite $\bar{\Theta}_{(n-3) / 2} \circ \cdots \circ \bar{\Theta}_{1}$. Any element of the right side is represented by $a_{1} \cdot U_{1}^{b} \cdot U_{2}^{p-1} \in C_{d-1-p(n-3) / 2}^{2}(X)$ for some $a_{1} \in \mathbb{Z}_{p}$ and $b \neq 0$. We will show $a_{1}=0$ as follows. By Lemma 3.9] it satisfies that

$$
a_{1}\left(\omega^{b}-1\right) \cdot U_{1}^{b}=\delta_{1}\left(a_{1} \cdot U_{1}^{b}\right)=a_{1}\left(\left(\omega U_{1}+U_{2}\right)^{b}-\left(U_{1}+U_{2}\right)^{b}\right) .
$$

By elementary calculation we have $b=1$ or $a_{1}=0$. If $a_{1} \neq 0$, then by comparing with the homogenous degrees, we have $1+(p-1)=d-1-p(n-3) / 2$, i.e., $d=p(n+1) / 2+1-p$, which implies $\omega^{d}=\omega^{(n+1) / 2} \neq 1$. Hence $a_{1}=0$, i.e., the right side in (23) vanishes.

Finally, we will show $c_{2 e-1}^{(0)}=1$. Note that it follows from Proposition 4.2 that the isomorphism given by (23) holds for the case of $n=2 e-1$ as well. Hence, it suffices to show that the right side in (23) is one dimensional. Note that if $d=e p-p+1$, then $\omega^{d}=1$. By equality (24) and the above discussion, the right side of (23) is generated by $U_{1} \cdot U_{2}^{p-1} \in C_{p}^{2}(X)$, which implies $c_{2 e-1}^{(0)} \leq 1$. We claim that $U_{1} \cdot U_{2}^{p-1} \in C_{p}^{2}(X)$ cannot be killed. Indeed the homogenous degree of any element in $B_{d-1-p(n-1) / 2}^{2}(X)$ is smaller than $p$ by definition. In the sequel we obtain $c_{2 e-1}^{(0)}=1$.

Remark 4.5. Since $\delta_{1}\left(U_{1}\right)=(\omega-1) \cdot U_{1}$ by definition, it follows from Corollary 4.4 that $H^{2 e-1(0)}(X) \cong \mathbb{Z}_{p}$ is spanned by

$$
U_{1} \cdot E_{2 \leq 2 e-3}^{\omega}\left(U_{2}, \ldots, U_{2 e-3}\right) \cdot E_{2 e-2}^{\omega}\left(U_{2 e-2}, U_{2 e-1}\right) \in C_{e p-p+1}^{2 e-1}(X) .
$$

In the case of $\omega=-1$ and $n=3$, the resulting 3 -cocycle $U_{1} \cdot E_{2}^{\omega}\left(U_{2}, U_{3}\right)$ is found in [M1, Theorem 0.3] and [M2, Example 2.4.1].

\section{Quandle Cocycles and the Proof of Theorem 2.1}

In this section we deal with $H^{n(0)}(X)$ for $n \geq 2 e$. For this, recall from Corollary 4.3 that $H^{n(0)}(X)$ is isomorphic to the quotient space on the right side of (21). In Section 5.1 we will present some examples of the cocycles and construct a homomorphism from $H^{n-2 e(0)}(X) \oplus H^{n-2 e+1(0)}(X) \oplus H^{n-2 e+2(0)}(X)$ to the quotient space. In Section 5.2 we will show that the map is isomorphic (Proposition 5.4). In Section 5.3 we will prove Theorem 3.3 and the main theorem 2.2 As a result, we will formulate presentations of all cocycles which span $H^{n}(X)$ (Corollary 5.8).

Throughout this section we fix $n \geq 2 e$ and $d$ satisfying $\omega^{d}=1$. Further, we denote by $e$ the order of $\omega$. To simplify the exposition, we define $d^{\prime}=d+(2-e) p$ and $m=n-2 e+4$. Note that $m \geq 4, \omega^{d^{\prime}-p-1}=\omega^{d^{\prime}-2 p}=1$ and $(-1)^{m}=(-1)^{n}$. Also notice that, if $\omega=-1$, then $d=d^{\prime}$ and $m=n$. 


\subsection{Examples of $n$-cocycles of the Alexander quandle of prime order.}

Example 5.1. Let $f_{m-3} \in C_{d^{\prime}-p-1}^{m-3}(X)$ be an $(m-3)$-cocycle which is divisible by $U_{m-3}$. Put $g_{p-1}\left(U_{1}, \ldots, U_{m-2}\right):=f_{m-3}\left(U_{1}, \ldots, U_{m-3}\right) \cdot U_{m-2}$. Then we can check $\delta_{m-2}\left(g_{p-1}\right)=(-1)^{m}(1-\omega) \cdot g_{p-1}$ using formula (3). According to Corollary 4.4, by applying such $g_{p-1}$ to (22), we have an $n$-cocycle given by

$f_{m-3}\left(U_{1}, \ldots, U_{m-3}\right) \cdot U_{m-2} \cdot E_{m-1 \leq n-2}^{\omega}\left(U_{m-1}, \ldots, U_{n-2}\right) \cdot E_{n-1}^{\omega}\left(U_{n-1}, T_{n}\right) \in C_{d}^{n}(X)$, where $E_{n-1}^{\omega}\left(U_{n-1}, T_{n}\right)$ is the polynomial given by (13).

Example 5.2. We define the following polynomial $F_{m-3}^{\omega}\left(U_{m-3}, U_{m-2}\right) \in$ $\mathbb{Z}_{p}\left[U_{m-3}, U_{m-2}\right]$ :

$$
\begin{aligned}
F_{m-3}^{\omega}\left(U_{m-3}, U_{m-2}\right) & =\left(\left(U_{m-3}+U_{m-2}\right)^{p}-\left(\omega U_{m-3}+U_{m-2}\right)^{p}+(\omega-1) U_{m-3}^{p}\right) / p \\
& \equiv \sum_{2 \leq j \leq p-1}(-1)^{j-1} \cdot j^{-1} \cdot\left(1-\omega^{1-j}\right) \cdot U_{m-3}^{p-j} \cdot U_{m-2}^{j}(\bmod p) .
\end{aligned}
$$

If $f_{m-4} \in C_{d^{\prime}-2 p}^{m-4}(X)$ is an $(m-4)$-cocycle with no term of $C_{d^{\prime}-2 p}^{m-4(1)}(X)$, then we put $g_{p-1}\left(U_{1}, \ldots, U_{m-2}\right):=f_{m-4}\left(U_{1}, \ldots, U_{m-4}\right) \cdot F_{m-3}^{\omega}$. Hence by direct calculation we have $\delta_{m-2}\left(g_{p-1}\right)=(-1)^{m}(1-\omega) \cdot g_{p-1}$, which satisfies the condition in Corollary 4.4. Thus, by applying such $g_{p-1}$ to (22), we obtain an $n$-cocycle given by

$$
\begin{aligned}
f_{m-4}\left(U_{1}, \ldots, U_{m-4}\right) & \cdot F_{m-3}^{\omega}\left(U_{m-3}, U_{m-2}\right) \\
& \cdot E_{m-1 \leq n-2}^{\omega}\left(U_{m-1}, \ldots, U_{n-2}\right) \cdot E_{n-1}^{\omega}\left(U_{n-1}, T_{n}\right) \in C_{d}^{n}(X) .
\end{aligned}
$$

For example, when $\omega=-1, n=4$, and $f_{0}=1$, we have a 4 -cocycle of the form

$$
\begin{aligned}
& \psi_{4,0}\left(U_{1}, U_{2}, U_{3}, T_{4}\right) \\
& \quad=\left(\left(U_{1}+U_{2}\right)^{p}+\left(U_{1}-U_{2}\right)^{p}-2 U_{1}^{p}\right) \cdot\left(\left(U_{3}+T_{4}\right)^{p}-\left(U_{3}-T_{4}\right)^{p}-2 T_{4}^{p}\right) / p^{2} .
\end{aligned}
$$

Example 5.3. We introduce the following polynomial $G_{m-3}^{\omega}\left(U_{m-3}, U_{m-2}\right) \in$ $\mathbb{Z}_{p}\left[U_{m-3}, U_{m-2}\right]$ :

$$
\begin{aligned}
G_{m-3}^{\omega}= & \left(\left(U_{m-3}+U_{m-2}\right)^{p+1}-\omega\left(U_{m-3}+\omega^{-1} U_{m-2}\right)^{p+1}\right. \\
& \left.-(1-\omega) U_{m-3}^{p+1}-\left(1-\omega^{-1}\right) U_{m-2}^{p+1}\right) / p \\
\equiv & \sum_{1 \leq j \leq p-2}(-1)^{j-1} \cdot j^{-1}(j+1)^{-1} \cdot\left(1-\omega^{-j}\right) \cdot U_{m-3}^{p-j} \cdot U_{m-2}^{j+1} \quad(\bmod p) .
\end{aligned}
$$

Let $f_{m-2}$ be an $(m-2)$-cocycle of the form

$$
f_{m-2}\left(U_{1}, \ldots, U_{m-2}\right)=f_{m-4}^{\prime}\left(U_{1}, \ldots, U_{m-4}\right) \cdot E_{m-3}^{\omega}\left(U_{m-3}, U_{m-2}\right) .
$$

Then we put $g_{p-1}:=f_{m-4}^{\prime} \cdot G_{m-3}^{\omega}$. By direct calculations we get $\delta_{m-2}\left(g_{p-1}\right)=$ $(-1)^{n}(1-\omega) \cdot g_{p-1}$. Therefore, because of (22) we find an $n$-cocycle given by

$$
\begin{aligned}
f_{m-4}^{\prime}\left(U_{1}, \ldots, U_{m-4}\right) & \cdot G_{m-3}^{\omega}\left(U_{m-3}, U_{m-2}\right) \\
& \cdot E_{m-1 \leq n-2}^{\omega}\left(U_{m-1}, \ldots, U_{n-2}\right) \cdot E_{n-1}^{\omega}\left(U_{n-1}, T_{n}\right) \in C_{d}^{n}(X) .
\end{aligned}
$$

For example, when $\omega=-1, n=5$, and $f_{3}^{\prime}=U_{1} \cdot E_{2}^{\omega}\left(U_{2}, U_{3}\right)$ is the above 3-cocycle presented in Remark 4.5, we have a 5 -cocycle given by

$U_{1} \cdot\left(\left(U_{2}+U_{3}\right)^{p+1}+\left(U_{2}-U_{3}\right)^{p+1}-2 U_{2}^{p+1}-2 U_{3}^{p+1}\right) \cdot\left(\left(U_{4}+T_{5}\right)^{p}-\left(U_{4}-T_{5}\right)^{p}-2 T_{5}^{p}\right) / p^{2}$. 
We will construct a map from $H^{m-2(0)}(X) \oplus H^{m-3(0)}(X) \oplus H^{m-4(0)}(X)$ given below in (29). Examples 5.1 5.2, and 5.3 enable us to define a homomorphism.

$$
\begin{gathered}
\Psi_{d^{\prime}}^{m}:\left(Z_{d^{\prime}-2 p}^{m-4}(X) / Z_{d^{\prime}-2 p}^{m-4(1)}(X)\right) \oplus\left(Z_{d^{\prime}-p-1}^{m-3}(X) / Z_{d^{\prime}-p-1}^{m-3(1)}(X)\right) \\
\oplus\left(Z_{d^{\prime}-p-1}^{m-2}(X) \cap C^{m-4}(X) \cdot E_{m-3}^{\omega}\right) \\
\longrightarrow \frac{\delta_{m-1}^{-1}\left(\operatorname{Im}\left(\mathcal{D}_{d^{\prime}}^{m}\right)\right)}{\operatorname{Im}\left(\mathcal{D}_{d^{\prime}}^{m-1}\right)+B_{d^{\prime}-1}^{m-1}(X)}, \\
\Psi_{d^{\prime}}^{m}\left(f_{m-4}, f_{m-3}, f_{m-4}^{\prime} \cdot E_{m-3}^{\omega}\right) \\
:=f_{m-4} \cdot F_{m-3}^{\omega} \cdot T_{m-1}^{p-1}+f_{m-3} \cdot U_{m-2} \cdot T_{m-1}^{p-1}+f_{m-4}^{\prime} \cdot G_{m-3}^{\omega} \cdot T_{m-1}^{p-1} .
\end{gathered}
$$

Furthermore, we extend the map on the third direct summand to a map on $Z_{d^{\prime}-p-1}^{m-2}(X)$ defined by zero outside $C^{m-4}(X) \cdot E_{m-3}^{\omega}$. By elementary calculation, we can check that the map does not depend on the coboundaries. We thus obtain the induced map as follows:

$$
\bar{\Psi}^{m}: H^{m-4(0)}(X) \oplus H^{m-3(0)}(X) \oplus H^{m-2(0)}(X) \longrightarrow \bigoplus_{d^{\prime}} \frac{\delta_{m-1}^{-1}\left(\operatorname{Im}\left(\mathcal{D}_{d^{\prime}}^{m}\right)\right)}{\operatorname{Im}\left(\mathcal{D}_{d^{\prime}}^{m-1}\right)+B_{d^{\prime}-1}^{m-1}(X)},
$$

where the direct sums are over all $d^{\prime}$ satisfying $\omega^{d^{\prime}}=\omega^{2}$, i.e., $\omega^{d}=1$. We will show

Proposition 5.4. Let $\omega^{d}=1$. The above map $\Psi_{d^{\prime}}^{m}$ is isomorphic. Hence, so is $\bar{\Psi}^{m}$.

5.2. The proof of Proposition 5.4. Since the proof is technical, the reader may skip it without loss of continuity.

For the proof, we need differential operations $p-1$ times and $p$ times: Let us consider the composite $\mathcal{D}_{d-j+1}^{n} \circ \mathcal{D}_{d-j+2}^{n} \circ \cdots \circ \mathcal{D}_{d}^{n}: C_{d}^{n}(X) \rightarrow C_{d-j}^{n}(X)$. We denote it by $\mathcal{D}_{d-j \leq d}^{n}$ for short. It goes without saying that $\mathcal{D}_{d-j \leq d}^{n}$ is a chain homomorphism, $\delta_{n} \circ \mathcal{D}_{d-j \leq d}^{n}=\mathcal{D}_{d-j \leq d}^{n+1} \circ \delta_{n}$. Also note that $\mathcal{D}_{d-p \leq d}^{n}(f)=0$ and that $\mathcal{D}_{d-p+1 \leq d}^{n}(f) \in C_{d-p+1}^{n(1)}(X)$ means the coefficient of $T_{n}^{p-1}$ in $-f$. Since $C_{d-p+1}^{n(1)}(X)$ injects $C_{d-p+1}^{n-1}(X)$ as shown in Proposition 3.2 , we often regard $\mathcal{D}_{d-p+1 \leq d}^{n}(f)$ as being in $C_{d-p+1}^{n-1}(X)$.

Proof. We will show the surjectivity of $\Psi_{d^{\prime}}^{m}$ in Step 1 and the injectivity in Step 2.

Step 1. Let $g$ be an element of the right side of (28). As usual, $g \in C_{d^{\prime}-1}^{m-1}(X)$ may satisfy $g\left(U_{1}, \ldots, U_{m-2}, T_{m-1}\right)=g_{p-1}\left(U_{1}, \ldots, U_{m-2}\right) \cdot T_{m-1}^{p-1}$ and $\delta_{m-1}(g) \in$ $\operatorname{Im}\left(\mathcal{D}_{d^{\prime}}^{m}\right)$. Then $g_{p-1}$ satisfies (12) by Lemma 3.9, which is rewritten in (30)

$\delta_{m-2}\left(g_{p-1}\right)\left(U_{1}, \ldots, U_{m-1}\right)=(-1)^{m}\left(1-\omega^{d^{\prime}-1}\right) \cdot g_{p-1}\left(U_{1}, \ldots, U_{m-2}\right) \in C_{d^{\prime}-p}^{m-1(1)}(X)$.

We will show equality (31) below. For this, taking $\mathcal{D}_{d^{\prime}-p}^{m-1}$ to (30), we obtain

$$
0=\mathcal{D}_{d^{\prime}-p}^{m-1}\left(\delta_{m-2}\left(g_{p-1}\right)\right)=\delta_{m-2}\left(\mathcal{D}_{d^{\prime}-p}^{m-2}\left(g_{p-1}\right)\right) .
$$

Namely, $\mathcal{D}_{d^{\prime}-p}^{m-2}\left(g_{p-1}\right) \in C_{d^{\prime}-p-1}^{m-2}$ is a cocycle. Recall here the decomposition $H_{d^{\prime}-p-1}^{m-2} \cong H_{d^{\prime}-p-1}^{m-2(0)} \oplus H_{d^{\prime}-p-1}^{m-3(0)}$ in Proposition 3.2 . Hence, there exist two representative cocycles $f_{m-2}$ of $H_{d^{\prime}-p-1}^{m-2(0)}(X)$ and $f_{m-3}$ of $H_{d^{\prime}-p-1}^{m-3(0)}(X)$ and a polynomial 
$h \in C_{d^{\prime}-p-1}^{m-3}(X)$ satisfying

$$
\mathcal{D}_{d^{\prime}-p}^{m-2}\left(g_{p-1}\right)=f_{m-2}+f_{m-3}+\delta_{m-3}(h) \in C_{d^{\prime}-p-1}^{m-2}(X) .
$$

Here, by Proposition [3.10, we may replace $f_{m-2}$ by a cocycle of the form $f_{m-4}^{\prime}$. $E_{m-3}^{\omega}$.

We focus on the third term $\delta_{m-3}(h)$ in (31) operated by the composite $\mathcal{D}_{d^{\prime}-2 p \leq d^{\prime}-p-1}^{m-2}$. Note that $\mathcal{D}_{d^{\prime}-2 p \leq d^{\prime}-p-1}^{m-2}$ annihilates $f_{m-4}^{\prime} \cdot E_{m-3}^{\omega}$ from the definition of $E_{m-3}^{\omega}$. Let $h_{d^{\prime}-2 p}^{m-4} \in C_{d^{\prime}-p-1}^{m-4}$ denote $\mathcal{D}_{d^{\prime}-2 p \leq d^{\prime}-p-1}^{m-3}(h)$. Hence, by applying $\mathcal{D}_{d^{\prime}-2 p \leq d^{\prime}-p-1}^{m-2}$ to (31), we have

$$
0=\delta_{m-3}\left(\mathcal{D}_{d^{\prime}-2 p \leq d^{\prime}-p-1}^{m-3}(h)\right)=\delta_{m-3}\left(h_{d^{\prime}-2 p}^{m-4} \cdot U_{m-3}^{0}\right)=\delta_{m-4}\left(h_{d^{\prime}-2 p}^{m-4}\right),
$$

where the third equality follows from (3) and $\omega^{d^{\prime}-2 p}=1$. Thus $-h_{d^{\prime}-2 p}^{m-4}$ equals some $(m-4)$-cocycle $f_{m-4}$. Hence $h=f_{m-4} \cdot U_{m-3}^{p-1}+\mathcal{D}_{d^{\prime}-p}^{m-3}\left(k^{\prime}\right)$ for some $k^{\prime} \in C_{d^{\prime}-p}^{m-3}(X)$. To summarize, equality (31) is rewritten as

$$
\mathcal{D}_{d^{\prime}-p}^{m-2}\left(g_{p-1}\right)=f_{m-4} \cdot E_{m-3}^{\omega}+f_{m-3}+\delta_{m-3}\left(f_{m-4} \cdot U_{m-3}^{p-1}\right)+\mathcal{D}_{d^{\prime}-p}^{m-2}\left(\delta_{m-3}\left(k^{\prime}\right)\right)
$$

Here we present the following two formulas obtained by direct calculations:

$$
\begin{gathered}
\mathcal{D}_{d^{\prime}-p+1}^{m-2}\left(f_{m-4} \cdot F_{m-3}^{\omega}\right)=\delta_{m-3}\left(f_{m-4} \cdot U_{m-3}^{p-1}\right), \\
\mathcal{D}_{d^{\prime}-p+1}^{m-2}\left(f_{m-4} \cdot G_{m-3}^{\omega}\right)=f_{m-4} \cdot E_{m-3}^{\omega} .
\end{gathered}
$$

Therefore, integrating (32) by $U_{m-2}$, we conclude

$$
g_{p-1}=f_{m-4} \cdot G_{m-3}^{\omega}+f_{m-3} \cdot U_{m-3}+f_{m-4}^{\prime} \cdot F_{m-3}^{\omega}+\delta_{m-3}\left(k^{\prime}\right) .
$$

Here, we claim $\delta_{m-3}\left(k^{\prime}\right)=0$. This is because that $g_{p-1}$ satisfies equality (30) above, and that, by the discussions in Examples 5.1, 5.2, and 5.3, we have known a similar equality

$$
\delta_{m-2}\left(g_{p-1}-\delta_{m-3}\left(k^{\prime}\right)\right)=(-1)^{m}\left(1-\omega^{d^{\prime}-1}\right)\left(g_{p-1}-\delta_{m-3}\left(k^{\prime}\right)\right) \in C_{d^{\prime}-p}^{m-1(1)}(X) .
$$

Since $g=g_{p-1} U_{m-1}^{p-1}$ by definition, (34) precisely means the surjectivity of $\Phi_{d^{\prime}}^{m}$.

Step 2. To prove the injectivity, for cocycles $f_{m-2}, f_{m-3}$, and $f_{m-4}^{\prime} \cdot E_{m-3}^{\omega}$, we let $h \in C_{d^{\prime}-1}^{m-2}$ and $k \in C_{d^{\prime}}^{m-1}$ satisfy

$$
-\left(f_{m-4} \cdot F_{m-3}^{\omega}+f_{m-3} \cdot U_{m-2}+f_{m-4}^{\prime} \cdot G_{m-3}^{\omega}\right) \cdot T_{m-1}^{p-1}=\delta_{m-2}(h)+\mathcal{D}_{d^{\prime}}^{m-1}(k) .
$$

Our purpose is to check that each of the cocycles are cohomologous to zero. By operating $\mathcal{D}_{d^{\prime}-p \leq d^{\prime}-1}^{m-1}$ to both the left and right sides, we have

$f_{m-4} \cdot F_{m-3}^{\omega}+f_{m-3} \cdot U_{m-2}+f_{m-4}^{\prime} \cdot G_{m-3}^{\omega}=\delta_{m-2}\left(\mathcal{D}_{d^{\prime}-p \leq d^{\prime}-1}^{m-2}(h)\right) \in C_{d^{\prime}-p}^{m-1(1)}(X)$.

The polynomial $\mathcal{D}_{d^{\prime}-p \leq d^{\prime}-1}^{m-2}(h) \in C_{d^{\prime}-p}^{m-2(1)}(X)$ may be regarded as that in $C_{d^{\prime}-p}^{m-3}(X)$. Let us denote it by $h^{\prime} \in C_{d^{\prime}-p}^{m-3}(X)$. Then, because of (3), we notice

$$
\begin{aligned}
\delta_{m-2}\left(\mathcal{D}_{d^{\prime}-p \leq d^{\prime}-1}^{m-2}(h)\right) & =\delta_{m-2}\left(h^{\prime} \cdot U_{m-2}^{0}\right) \\
& =\delta_{m-3}\left(h^{\prime}\right)+(-1)^{m}\left(1-\omega^{-1}\right) \cdot h^{\prime} \in C_{d^{\prime}-p}^{m-2(1)}(X) .
\end{aligned}
$$

Also notice $\mathcal{D}_{d^{\prime}-p}^{m-2}\left(h^{\prime} \cdot U_{m-2}^{0}\right)=0$. Thereby, by applying $\mathcal{D}_{d^{\prime}-p}^{m-2}$ to (35),

(36) $\mathcal{D}_{d^{\prime}-p}^{m-2}\left(f_{m-4} \cdot F_{m-3}^{\omega}\right)+f_{m-3}+f_{m-4}^{\prime} \cdot E_{m-3}^{\omega}=\delta_{m-3}\left(\mathcal{D}_{d^{\prime}-p}^{m-3}\left(h^{\prime}\right)\right) \in C_{d^{\prime}-p-1}^{m-2}(X)$. 
Recall from (33) that $\mathcal{D}_{d^{\prime}-p+1}^{m-2}\left(f_{m-4} \cdot F_{m-3}^{\omega}\right)=\delta_{m-3}\left(f_{m-4} \cdot U_{m-3}^{p-1}\right)$. Therefore,

$$
f_{m-3}+f_{m-4}^{\prime} \cdot E_{m-3}^{\omega}=\delta_{m-3}\left(\mathcal{D}_{d^{\prime}-p}^{m-3}\left(h^{\prime}\right)-f_{m-4} \cdot U_{m-3}^{p-1}\right) .
$$

Namely, it follows from Proposition 3.2 (I) that $f_{m-3}$ and $f_{m-4}^{\prime} \cdot E_{m-3}^{\omega}$ are nullcohomologous.

Finally, it suffices to show that $f_{m-4}$ is cohomologous to zero. To begin, by applying $\mathcal{D}_{d^{\prime}-2 p+1 \leq d^{\prime}-p-1}^{m-2}$ to (36), from the definitions of $E_{m-3}^{\omega}$ and $F_{m-3}^{\omega}$, we have

$(1-\omega) f_{m-4} \cdot U_{m-3}+\frac{1-\omega^{2}}{2} f_{m-4}^{\prime} \cdot U_{m-3}^{2}=\delta_{m-3}\left(\mathcal{D}_{d^{\prime}-2 p+1 \leq d^{\prime}-p}^{m-3}\left(h^{\prime}\right)\right) \in C_{d^{\prime}-2 p+1}^{m-2(1)}(X)$.

Similarly to $\mathcal{D}_{d^{\prime}-p \leq d^{\prime}-1}^{m-2}(h)$ above, $\mathcal{D}_{d^{\prime}-2 p+1 \leq d^{\prime}-p}^{m-3}\left(h^{\prime}\right) \in C_{d^{\prime}-2 p+1}^{m-3(1)}$ may be contained in $C_{d^{\prime}-2 p+1}^{m-4}$. We denote it by $h^{\prime \prime}$. Then the right side of (38) is expanded to

$$
=\delta_{m-3}\left(h^{\prime \prime} \cdot U_{m-3}^{0}\right)=\delta_{m-4}\left(h^{\prime \prime}\right)+(-1)^{m}\left(1-\omega^{-1}\right) \cdot h^{\prime \prime} \in C_{d^{\prime}-2 p+1}^{m-2(1)}(X) .
$$

Hence, equality (38) sent by the operation $\mathcal{D}_{d^{\prime}-2 p+1}^{m-3}$ is reduced to

$$
(1-\omega) f_{m-4}+\left(1-\omega^{2}\right) f_{m-4}^{\prime} \cdot U_{m-3}=\delta_{m-4}\left(\mathcal{D}_{d^{\prime}-2 p+1}^{m-4}\left(h^{\prime \prime}\right)\right) \in C_{d^{\prime}-2 p}^{m-3}(X) .
$$

Therefore, since $\omega^{d^{\prime}-2 p}=1$, it follows from the decomposition $H_{d^{\prime}-2 p}^{m-3} \cong H_{d^{\prime}-2 p}^{m-3(0)} \oplus$ $H_{d^{\prime}-2 p}^{m-4(0)}$ in Proposition 3.2 that the cocycle $f_{m-4}$ is null-cohomologous.

5.3. The proofs of Theorems 3.3 (II) and 2.1. We now prove Theorem 3.3 (II), and, using it, we show Theorem 2.1.

Proof of Theorem 3.3(II). Recall the isomorphisms $\bar{\Theta}_{i}$ and $\bar{\Psi}_{d^{\prime}}^{m}$ in Propositions 4.2 and 5.4. By composing them as mentioned in Section 2.3, we have an isomorphism from $H^{n(0)}(X) \oplus H^{n+1(0)}(X) \oplus H^{n+2(0)}(X)$ to $H^{n+2 e(0)}(X)$, which immediately implies Theorem 3.3 (II).

Proof of Theorem 2.1. By Corollary 6.4 (which we will show later) $H_{n}^{Q}(X ; \mathbb{Z})$ is annihilated by $p$; that is, $H_{n}^{Q}(X ; \mathbb{Z})$ is a $\mathbb{Z}_{p}$-vector space. We thus have $H_{n}^{Q_{U}}(X ; \mathbb{Z}) \cong$ $\mathbb{Z}_{p}^{b_{n}}$ for some $b_{n}$. Recall Theorem 3.3(II): the dimension $c_{n}^{(0)}=\operatorname{dim}\left(H^{n(0)}\right)$ is determined by $c_{n+2 e}^{(0)}=c_{n+2}^{(0)}+c_{n+1}^{(0)}+c_{n}^{(0)}, c_{1}^{(0)}=\cdots=c_{2 e-2}^{(0)}=0, \quad$ and $c_{2 e-1}^{(0)}=c_{2 e}^{(0)}=1$. By Lemma 5.5 below, $b_{n}=c_{n}^{(0)}$ for $n \geq 2$. Hence, we obtain the recurrence formula in Theorem 2.2.

Lemma 5.5. The above $b_{n}$ satisfies $b_{n}=c_{n}^{(0)}$ for $n \geq 2$.

Proof. To begin, if $n=2$, it is shown [M1, Corollary 2.2] that $H_{Q}^{2}\left(X ; \mathbb{Z}_{p}\right) \cong 0$. By (2) we obtain $H_{2}^{Q_{U}}(X ; \mathbb{Z}) \cong 0$. Hence $b_{2}=c_{2}^{(0)}=0$. Furthermore, for $k>2$, we have $b_{k}+b_{k-1}=\operatorname{dim}\left(H^{k}(X)\right)=c_{k}^{(0)}+c_{k-1}^{(0)}$ by the universal coefficient theorem and Proposition 3.2. Hence, the induction on $k$ concludes $b_{n}=c_{n}^{(0)}$ for $n \geq 2$. 
5.4. Cohomological operations and presentations of $n$-cocycles. As a result of Theorem 3.3, we will present all cocycles which span the cohomology $H^{n}(X)$. To see this, we use the four polynomials $E_{n-1}^{\omega}, E_{n-2 e+3 \leq n-2}^{\omega}, F_{n-2 e+1}^{\omega}$, and $G_{n-2 e+1}^{\omega}$; see the formulas (13), (20), (26), and (27) for the definitions, respectively.

For this purpose, we now set up an operation on the quandle cohomology group (cf. [NP Sections 2 and 6] for a study on homological operations for certain quandles).

$$
\begin{gathered}
\Omega_{m}:\left(Z_{d^{\prime}-2 p}^{m-4}(X) / Z_{d^{\prime}-2 p}^{m-4(1)}(X)\right) \oplus\left(Z_{d^{\prime}-p-1}^{m-3}(X) / Z_{d^{\prime}-p-1}^{m-3(1)}(X)\right) \\
\quad\left(Z_{d^{\prime}-p-1}^{m-2}(X) \cap C^{m-4}(X) \cdot E_{m-3}^{\omega}\right) \\
\longrightarrow H_{d}^{n(0)}(X), \\
\Omega_{m}\left(f_{m-4}, f_{m-3}, f_{m-4}^{\prime} \cdot E_{m-3}^{\omega}\right) \\
:=\left(f_{m-4} \cdot F_{m-3}^{\omega}+f_{m-3} \cdot U_{m-2}+f_{m-4}^{\prime} \cdot G_{m-3}^{\omega}\right) \cdot E_{m+1 \leq n-2}^{\omega} \cdot E_{n-1}^{\omega},
\end{gathered}
$$

where $m=n-2 e+4$. We notice that this does not depend on the coboundaries by Propositions 3.7, 4.2 and 5.4. Therefore, the $\Omega_{m}$ induces

$$
\bar{\Omega}_{n-2 e+4}: H^{n-2 e(0)}(X) \oplus H^{n-2 e+1(0)}(X) \oplus H^{n-2 e+2(0)}(X) \longrightarrow H^{n(0)}(X) .
$$

From the construction, we can check that by Proposition 3.7 and Corollary 4.4 the above $\bar{\Omega}_{n-2 e+4}$ is equal to the composite $\bar{\phi}^{-1} \circ\left(\bar{\Theta}_{1}\right)^{-1} \circ \ldots \circ\left(\bar{\Theta}_{e-1}\right)^{-1} \circ \bar{\Psi}^{m}$. Since each homomorphism is an isomorphism, we thus obtain

Corollary 5.6. $\bar{\Omega}_{n-2 e+4}$ is an isomorphism for $n \geq 2 e$.

Remark 5.7. Recall $H^{n}(X) \cong H^{n(0)}(X) \oplus H^{n-1(0)}(X)$ from Proposition 3.2. Then, the direct sum $\bar{\Omega}_{n-2 e+4} \oplus \bar{\Omega}_{n-2 e+3}$ induces an isomorphism on the quandle cohomology $H^{n}(X)$.

Next, we will present all cocycles which span the cohomology $H^{n}(X)$. For this purpose we will define sets of some $n$-cocycles denoted by $\operatorname{Coc}_{n}^{\omega,(0)}$ as follows. We first define $\operatorname{Coc}_{0}^{\omega,(0)}$ to be $1 \in \mathbb{Z}_{p}$. Define $\operatorname{Coc}_{i}^{\omega,(0)}$ by the empty sets for $0<i<$ $2 e-1$. Put $\operatorname{Coc}_{2 e-1}^{\omega,(0)}:=\left\{U_{1} \cdot E_{2 \leq 2 e-3}^{\omega} \cdot E_{2 e-2}^{\omega} \in C^{2 e-1}(X)\right\}$. By induction on $n$ we define $\operatorname{Coc}_{n}^{\omega,(0)} \subset C^{n}(X)$ as follows:

$\operatorname{Coc}_{n}^{\omega,(0)}$

$$
\begin{aligned}
& :=\left\{f_{n-2 e} \cdot F_{n-2 e+1}^{\omega} \cdot E_{n-2 e+3 \leq n-2}^{\omega} \cdot E_{n-1}^{\omega} \mid f_{n-2 e} \in \operatorname{Coc}_{n-2 e}^{\omega,(0)}\right\} \\
& \cup\left\{f_{n-2 e+1} \cdot U_{n-2 e+2} \cdot E_{n-2 e+3 \leq n-2}^{\omega} \cdot E_{n-1}^{\omega} \mid f_{n-2 e+1} \in \operatorname{Coc}_{n-2 e+1}^{\omega,(0)}\right\} \\
& \cup\left\{f_{n-2 e+2} \cdot G_{n-2 e+1}^{\omega} \cdot E_{n-2 e+3 \leq n-2}^{\omega} \cdot E_{n-1}^{\omega} / E_{n-2 e+1}^{\omega} \mid f_{n-2 e+2} \in \operatorname{Coc}_{n-2 e+2}^{\omega,(0)}\right\},
\end{aligned}
$$

noting that $f_{n-2 e+2} / E_{n-2 e+1}^{\omega} \in C^{n-2 e}(X)$ from the definition of $\operatorname{Coc}_{n-2 e+2}^{\omega,(0)}$. Also, by definition, notice that any element of $\operatorname{Coc}_{n}^{\omega,(0)}$ has no term of $C^{n(1)}(X)$.

Corollary 5.8. Let $X$ be the Alexander quandle of order $p$ with $\omega \neq 0,1$. Then $H^{n(0)}(X)$ is independently generated by $\operatorname{Coc}_{n}^{\omega,(0)}$. Further, the quandle cohomology $H^{n}(X)$ is independently generated by $\operatorname{Coc}_{n}^{\omega,(0)} \cup \operatorname{Coc}_{n-1}^{\omega,(0)}$, where we regard the $(n-1)$ cocycles in $\operatorname{Coc}_{n-1}^{\omega,(0)}$ as n-cocycles of $C^{n(1)}(X)$. 
Proof. By definition $H^{0}(X) \cong \mathbb{Z}_{p}$ is generated by $1 \in \mathbb{Z}_{p}$. By Theorem 3.3 we have $H^{i(0)}(X) \cong 0$ for $0<i<2 e-1$. By Remark 4.5, $H^{2 e-1(0)}(X) \cong \mathbb{Z}_{p}$ is spanned by $\operatorname{Coc}_{2 e-1}^{\omega,(0)}$. Let us consider the case $n \geq 2 e$. Assume that, for $n \leq k-1, H^{n(0)}(X)$ is independently generated by $\operatorname{Coc}_{n}^{\omega,(0)}$. Note that the construction of $\operatorname{Coc}_{k}^{\omega,(0)}$ is compatible with the isomorphism $\bar{\Omega}_{k-2 e+4}$. Therefore, $H^{k(0)}(X)$ is independently generated by $\operatorname{Coc}_{k}^{\omega,(0)}$.

To show the latter part, recall from Proposition 3.2(II) that a canonical inclusion $C^{n(1)}(X) \subset C^{n-1}(X)$ induces $H^{n(1)}(X) \cong H^{n-1(0)}(X)$. Therefore $H^{n(1)}(X)$ is spanned by $\operatorname{Coc}_{n-1}^{\omega,(0)}$. Moreover by the direct sum decomposition $H^{n}(X) \cong$ $H^{n(0)}(X) \oplus H^{n(1)}(X), H^{n}(X)$ is independently generated by $\operatorname{Coc}_{n}^{\omega,(0)} \cup \operatorname{Coc}_{n-1}^{\omega,(0)}$.

Finally, we end this section by taking examples of 4-cocycles.

Example 5.9. Let $\omega=-1$ and $n=4$. Then $H_{Q}^{4}\left(X ; \mathbb{Z}_{p}\right) \cong\left(\mathbb{Z}_{p}\right)^{2}$. Further, the two bases are represented by

$$
\begin{aligned}
\psi_{4,0}(x, y, z, w) & :=(x-y) \cdot\left(2(z-w)^{p}-(2 z-w-y)^{p}-(y-w)^{p}\right) / p, \\
\psi_{4,1}(x, y, z, w):=\left((x-2 y+z)^{p}+(x-z)^{p}-2(x-y)^{p}\right) & \cdot\left(2 w^{p}-(2 z-w)^{p}-z^{p}\right) / p^{2} .
\end{aligned}
$$

Here, we use the usual coordinate system of $C_{Q}^{4}\left(X ; \mathbb{Z}_{p}\right)$, such as in CJKLS, CKS].

\section{TORSION SUBgroup OF INTEGRAL QUANDLE HOMOLOGY}

The goal in this section is to show Theorem 6.1. As a corollary, for an Alexander quandle $X$ of order $p$, the quandle homology is annihilated by $p$ (Corollary 6.4).

We will review the finite connected Alexander quandles and the torsion subgroups of the quandle homology groups. Let $M$ be an Alexander quandle, that is, $M$ is a $\mathbb{Z}\left[T, T^{-1}\right]$-module with a binary operation given by $x * y=T x+(1-T) y$. If the quotient module $M /(1-T) M$ is zero, the quandle is said to be connected. It is known [LN, Proposition 1] that this definition is equivalent to the standard definition of the connectivity. R. A. Litherland and S. Nelson showed in [LN, Theorem 1] that if $M$ is finite and connected, then the free subgroup of $H_{n}^{R}(M ; \mathbb{Z})$ is $\mathbb{Z}$ and the generator is represented by $(0,0, \ldots, 0) \in C_{n}^{R}(M ; \mathbb{Z})$ and that the torsion subgroup is annihilated by $|M|^{n}$ for each $n \geq 1$. It is shown in [NP, Theorem 17] that for the dihedral quandle $X$ of order 3 , the torsion part of $H_{n}^{R}(X ; \mathbb{Z})$ is annihilated by 3 .

More generally, in this paper we will show a stronger estimate of the integral quandle homology group for finite connected Alexander quandles.

Theorem 6.1. Let $M$ be a finite connected Alexander quandle. Then, for each $n$, the torsion part of $H_{n}^{R}(M ; \mathbb{Z})$ is annihilated by $|M|$.

Before the proof, we will present some corollaries and remarks.

Corollary 6.2. Let $M$ be as above. Then $H_{n}^{Q}(M ; \mathbb{Z})$ is annihilated by $|M|$ for $n \geq 2$.

Proof. According to $[\mathrm{LN}$, Theorem 4$], H_{n}^{Q}(M ; \mathbb{Z})$ is shown to be a direct summand of $H_{n}^{R}(M ; \mathbb{Z})$. Since $(0, \ldots, 0)$ is zero in $C_{n}^{Q}(M ; \mathbb{Z}), H_{n}^{Q}(M ; \mathbb{Z})$ has no free part. 
Remark 6.3. It is false that in general, for a finite connected quandle, $H_{n}^{Q}(X ; \mathbb{Z})$ is annihilated by $|X|$. For example, let $Q S(6)$ be the connected quandle of order 6 presented by CKS, Example 2.2]. $Q S(6)$ is not isomorphic to any Alexander quandle (see [O, Section 5.1]). Then it is known [CKS, Example 2.5] that $H_{3}^{Q}(Q S(6) ; \mathbb{Z}) \cong \mathbb{Z} / 24 \mathbb{Z} \cong \mathbb{Z} / 2^{3} \mathbb{Z} \oplus \mathbb{Z} / 3 \mathbb{Z}$.

We immediately obtain the following corollaries, which we state without proof.

Corollary 6.4. Let $X$ be an Alexander quandle of order $p$, that is, $X=$ $\mathbb{Z}_{p}[T] /(T-\omega)$ for some $\omega \neq 0,1$. Then $H_{n}^{Q}(X ; \mathbb{Z})$ is annihilated by $p$.

Corollary 6.5. If $X=\mathbb{Z}_{2}[T] /\left(T^{2}+T+1\right)$ with the Alexander quandle operation, then $H_{n}^{Q}(X ; \mathbb{Z})$ is annihilated by 4 .

Remark 6.6. Corollary 6.4 is conjectured in [NP, Conjecture 16].

Proof of Theorem 6.1. We will work with our coordinate system of the rack complex in (1). We now introduce two chain maps $f_{+}^{j}, f_{0}^{j}: C_{n}^{R_{U}}(M ; \mathbb{Z}) \rightarrow C_{n}^{R_{U}}(M ; \mathbb{Z})$ given by

$$
\begin{gathered}
f_{+}^{j}\left(U_{1}, \ldots, U_{n}\right)= \begin{cases}\sum_{y \in M}\left(0, \ldots, 0, y, U_{j+1}, \ldots, U_{n}\right) & \text { for } 0<j<n \\
\sum_{y \in M}(0, \ldots, 0, y) & \text { for } j=n,\end{cases} \\
f_{0}^{j}\left(U_{1}, \ldots, U_{n}\right)= \begin{cases}|M| \cdot\left(0, \ldots, 0, U_{j}, U_{j+1}, \ldots, U_{n}\right) & \text { for } 0<j<n \\
|M| \cdot(0, \ldots, 0) & \text { for } j=n .\end{cases}
\end{gathered}
$$

Lemma 6.7. $f_{0}^{j}-f_{+}^{j}$ and $f_{0}^{j}-f_{+}^{j-1}$ are null-homotopic for any $j \leq n$.

We will show this below. If we assume the lemma, then $f_{0}^{1}=|M| \cdot \operatorname{id}_{C_{n}^{R_{U}}(M ; \mathbb{Z})}$ is chain homotopic to $f_{0}^{n}$. Hence we have the induced maps $\left(f_{0}^{1}\right)_{*}=\left(f_{0}^{n}\right)_{*}$ : $H_{n}^{R_{U}}(M ; \mathbb{Z}) \rightarrow H_{n}^{R_{U}}(M ; \mathbb{Z})$. However, recall that the free subgroup of $H_{n}^{R_{U}}(M ; \mathbb{Z})$ is generated by $(0, \ldots, 0)$ as mentioned above. Hence the image of $|M| \cdot \operatorname{id}_{H_{n}^{R_{U}}(M ; \mathbb{Z})}$ is contained in the free part of $H_{n}^{R_{U}}(M ; \mathbb{Z})$. Thus the torsion part of $H_{n}^{R_{U}}(M ; \mathbb{Z})$ is annihilated by $|M|$.

Proof of Lemma 6.7. By the connectivity, we recall $M=(1-T) M$ as a $\mathbb{Z}\left[T^{ \pm}\right]$module. Denote by $(1-T)^{-1}$ the inverse map of $1-T: M \rightarrow M$. Here we claim that chain homotopies $D_{n, 0}^{j}: C_{n}^{R_{U}}(M ; \mathbb{Z}) \rightarrow C_{n+1}^{R_{U}}(M ; \mathbb{Z})$ between $f_{0}^{j}$ and $f_{+}^{j}$ are given by the formula

$$
D_{n, 0}^{j}\left(U_{1}, \ldots, U_{n}\right)=\sum_{y \in M}\left(0, \ldots, 0,(T-1)^{-1} y, U_{j}+(1-T)^{-1} y, U_{j+1}, \ldots, U_{n}\right) .
$$

Indeed, a direct calculation verifies that $\partial_{n+1} D_{n, 0}^{j}+D_{n-1,0}^{j} \partial_{n}=(-1)^{j}\left(f_{+}^{j}-f_{0}^{j}\right)$.

Next, we put homomorphisms $D_{n,+}^{j}: C_{n}^{R_{U}}(M ; \mathbb{Z}) \rightarrow C_{n+1}^{R_{U}}(M ; \mathbb{Z})$ given by the formula

$$
\begin{aligned}
D_{n,+}^{j}( & \left.U_{1}, \ldots, U_{n}\right) \\
= & \begin{cases}\sum_{y \in M}\left(0, \ldots, 0,(1-T)^{-1} y,(T-1)^{-1} y, U_{j+1}, \ldots, U_{n}\right) & \text { for } j<n \\
\sum_{y \in M}\left(0, \ldots, 0,(1-T)^{-1} y,(T-1)^{-1} y\right) & \text { for } j=n .\end{cases}
\end{aligned}
$$


By direct calculation we obtain $\partial_{n+1} D_{n,+}^{j}+D_{n-1,+}^{j} \partial_{n}=(-1)^{j}\left(f_{+}^{j-1}-f_{0}^{j}\right)$ for $j>1$. Therefore $D_{n,+}^{j}$ are chain homotopies between $f_{+}^{j}$ and $f_{0}^{j+1}$. This completes the proof.

\section{ACKNOWLEDGMENTS}

The author thanks Toshiyuki Akita, Maciej Niebrzydowski, Józef Przytycki, Masahico Saito, Shin Satoh, Kokoro Tanaka, Michihisa Wakui, and Tadayuki Watanabe for valuable comments and discussions. He is sincerely grateful to Takuro Mochizuki for carefully reading the paper and making suggestions for its improvement. He also expresses his gratitude to Tomotada Ohtsuki for encouragement and useful advice.

\section{REFERENCES}

[AS] S. Asami, S. Satoh, An infinite family of non-invertible surfaces in 4-space, Bull. London Math. Soc. 37 (2005), 285-296. MR.2119028 (2005k:57044)

[CJKLS] J. S. Carter, D. Jelsovsky, S. Kamada, L. Langford, M. Saito, Quandle cohomology and state-sum invariants of knotted curves and surfaces, Trans. Amer. Math. Soc. 355 (2003) 3947-3989. MR.1990571 (2005b:57048)

[CKS] J. S. Carter, S. Kamada, M. Saito, Geometric interpretations of quandle homology, J. Knot Theory Ramifications 10 (2001) 345-386. MR.1825963 (2002h:57009)

[Cla] F. J.-B. J. Clauwens, The algebra of rack and quandle cohomology, arXiv:math/1004. 4423.

[EGS] P. Etingof, R. Guralnick, A. Soloviev, Indecomposable set-theoretical solutions to the quantum Yang-Baxter equation on a set with a prime number of elements, J. Algebra 242 (2001) 709-719. MR.1848966 (2002e:20049)

[FRS] R. Fenn, C. Rourke, B. Sanderson, Trunks and classifying spaces, Appl. Categ. Structures 3 (1995) 321-356. MR.1364012 (96i:57023)

[LN] R. A. Litherland, S. Nelson, The Betti numbers of some finite racks, J. Pure Appl. Algebra 178, 2003, 187-202. MR1952425 (2004a:57006)

[M1] T. Mochizuki, Some calculations of cohomology groups of finite Alexander quandles, J. Pure Appl. Algebra 179 (2003) 287-330. MR.1960136 (2004b:55013)

[M2] The 3-cocycles of the Alexander quandles $\mathbb{F}_{q}[T] /(T-\omega)$, Algebraic and Geometric Topology. 5 (2005) 183-205. MR2135551 (2006e:57019)

[No] T. Nosaka, Quandle homotopy invariants of knotted surfaces, arXiv:math/1011. 6035.

[NP] M. Niebrzydowski, J. H. Przytycki. Homology of dihedral quandles, J. Pure Appl. Algebra 213 (2009) 742-755. MR2494367 (2010a:18014)

[O] T. Ohtsuki (ed.), Problems on invariants of knots and 3-manifolds, Geom. Topol. Monogr., 4, Invariants of knots and 3-manifolds (Kyoto, 2001), 377-572, Geom. Topol. Publ., Coventry, 2002. MR2065029(2005c:57014)

Research Institute for Mathematical Sciences, Kyoto University, Sakyo-ku, Kyoto, 606-8502, JAPAN

E-mail address: nosaka@kurims.kyoto-u.ac.jp 